\title{
Free energy and kinetics of cAMP permeation through connexin26 hemichannel with and without voltage
}

1. College of Pharmacy, Western University of Health Sciences, 309 E. Second St, Pomona, CA, USA.

2. Department of Pharmacology, Physiology, and Neuroscience. New Jersey Medical School, Rutgers, The State University of New Jersey, Newark, NJ, USA.

3. Department of Life and Environmental Sciences, Polytechnic University of Marche, Ancona, Italy

4. Center for Synaptic Neuroscience and Technology, Italian Institute of Technology, Genoa, Italy.

\# contribute equally

* corresponding authors

\section{Abstract}

18 The connexin family is a diverse group of highly regulated non- $\beta$-barrel wide-pore channels permeable to biological signaling molecules. Despite their critical roles in mediating selective molecular signaling in health and disease, the molecular basis of permeation through these pores remains unclear. Here, we report the thermodynamics and kinetics of binding and transport of a second messenger, adenosine-3',5'cyclophosphate (cAMP), through a connexin26 hemichannel. Inward and outward fluxes of cAMP were first obtained from $4 \mu$ s simulations with voltages and multiple cAMPs in solution. The results are compared with the intrinsic potential of mean force (PMF) and the mean first passage times (MFPTs) of a single cAMP in the absence of voltage, obtained from a total of $16.5 \mu \mathrm{s}$ of multi-replica Voronoitessellated Markovian milestoning simulations. The computed transit times through the pore correspond well to existing experimental data. Both voltage simulations and milestoning simulations revealed two cAMP binding sites with binding constants and dissociation rates computed from PMF and MFPTs. The protein dipole inside the pore produces an asymmetric PMF, reflected in unequal cAMP MFPTs in each direction once within the pore. The free energy profiles under voltages derived from intrinsic PMF provided a unified understanding of directional transition rates with/without voltage, and revealed the unique role of channel polarity and the mobile electrolyte within a wide pore on the total free energy. In addition, we show how these factors influence the cAMP dipole vector during permeation, and how cAMP affects the local and non-local pore diameter in a position-dependent manner. 


\section{Significance Statement}

38 Connexins are wide-pore channels permeable to cellular signaling molecules. They mediate molecular

39 signaling crucial in physiology, pathology, and development; mutations in connexins cause human

40 pathologies. However, the fundamental structural, thermodynamic, and kinetic determinants of molecular

41 permeability properties are unknown. Using multiple molecular dynamics simulation techniques, we

42 report, for the first time, an in-depth investigation of the free energy and the directional transition rates of

43 an important biological signaling molecule, cAMP, through a connexin channel. We reveal the energetics

44 and binding sites that determine the cAMP flux, and the effects of mobile ions and external electrical field

45 on the process. The results provide a basis for understanding the unique features of molecular flux through

46 connexins and other non- $\beta$-barrel wide-pore channels.

\section{Introduction}

50 Connexin proteins form wide channels that mediate electrical and molecular signaling through cell 51 membranes. They can function as plasma membrane channels (called "hemichannels) or as intercellular channels that allow direct transfer of small cytoplasmic molecules between cells. The

53 intercellular channels ("gap junction channels") are formed by end-to-end docking of two

54 hemichannels across the extracellular gap between adjacent cells. The pores are relatively wide and are therefore permeable to atomic ions and small molecules in the size-range of key cellular signaling molecules including cAMP, cGMP, ATP, $\mathrm{IP}_{3}$, and glutathione. Each of the 21 human connexin isoform forms channels with distinct permeability and regulatory properties $(1,2)$. With the exception of electrical signaling in excitable tissues, the primary biological function of connexin channels is to mediate movement of small cytoplasmic signaling molecules between cells and/or to release them into the extracellular environment in a highly regulated manner. Mutations

61 in connexins that alter channel function or expression produce human pathologies(3, 4). These

62 mutations ultimately exert their pathological effects by disrupting the proper molecular

63 permeability of junctional and plasma membranes that connexin channels mediate.

A large literature documents that channels formed by the different connexin isoforms have

65 dramatically different permeability properties. Their unitary conductances range from $10 \mathrm{pS}$ to

$66300 \mathrm{pS}$, their cation/anion permeability ratios $\left(P_{\mathrm{K}+} / P_{\mathrm{Cl}-}\right)$ range from 8.0 to 0.8 and their 
67 permeabilities to fluorescent tracers are highly disparate. Strikingly, none of these parameters 68 correlate with each other (e.g., the connexin channel with the largest unitary conductance is among 69 the most size-restrictive). Permeabilities to biological signaling molecules are strikingly different 70 among the different connexin channels, but are difficult to measure quantitatively, as the molecules 71 are not fluorescent and do not carry significant current. Furthermore, for a given type of connexin 72 channel, there are remarkable degrees of selectivity and relative permeability among biological 73 permeants. These connexin-specific and permeant-specific permeability properties are not 74 reasonably inferred from differences in permeabilities to fluorescent tracers(5). This suggests that 75 there are, as yet unknown, structural/energetic determinants of molecular permeation that impart 76 to each type of connexin channel specific, biologically required permeability properties. The 77 underlying mechanisms for this are unknown, in spite of their clear biomedical and therapeutic importance. Investigation of these mechanisms by mutagenesis has not been informative in the absence of an understanding of the behavior, energetics, and interactions experienced by a 80 molecule as it traverses the pore. Computational studies can provide the basis for this understanding.

We previously used Hamiltonian replica-exchange umbrella sampling to explore the energetics of uncharged permeant and non-permeant tracer molecules in the connexin 26 (Cx26)

84 hemichannel( 6 ). That study indicated that the determinants of molecular permeation differ from

85 those that dominate the permeation of atomic ions through commonly studied ion-selective 86 channels, emphasizing the unique aspects of small molecules with conformational and 87 orientational degrees of freedom in a wide pore. The free energy and calculated relative 88 permeabilities derived from that work were consistent with experimental findings. However, while 89 validating the atomistic system and overall computational approach, the previous work did not 90 provide quantitative kinetic information of the permeation process. Furthermore, the results of 91 neutral tracer molecules do not characterize the biomedically crucial process of permeation by 92 charged biological signaling molecules. The present study explores the binding and transport 93 kinetics of a negatively charged second messenger, adenosine-3',5'-cyclophosphate (cAMP), 94 permeates a connexin pore.

95 A single permeation event with a timescale of sub-microsecond to microseconds is within 96 reach of today's computational power. However, a large number of transition events are required 97 to obtain meaningful statistics, for which simulations of orders of magnitude longer than the mean 
transition time are required. One solution is to accelerate the permeation of charged molecules by imposing a voltage (cAMP carries a charge of -1e). Theoretically, if the system reaches a steady state under a constant electric field and maintains symmetric concentration on both sides of the channel, a mean flux rate can be estimated from the ensemble of nonequilibrium processes using

102 a large number of permeation events. The accumulated density of cAMP along the channel axis

103 during these events may provide an estimate of the locations of the energetic barrier(s) and binding 104 site(s). Alternatively, one can choose an enhanced sampling method that is suitable for computing 105 free energy and kinetics of the permeation process. In this study, we used both approaches to gain 106 a comprehensive understanding of cAMP permeation with and without voltage, and in presence 107 and absence of multiple permeants.

A molecular permeation rate is often dominated by the free energy profile or potential of mean force (PMF) along the channel lumen axis. Previously we estimated the relative transition

110 rates between two sugar molecules using transition state theory (TST) based on PMF profiles( $\sigma$ ).

111 However, TST requires assumptions such as a single dominant transition state and no re-crossing 112 at the barriers, which are often difficult to satisfy in complex biomolecular systems with rugged 113 free-energy landscapes. To overcome this limitation, enhanced sampling techniques have been 114 developed to calculate transition rates from molecular dynamics (MD) simulations. Of particular 115 interest is the milestoning method introduced by Faradjian and Elber(7), which has been developed 116 into several versions and used in many biophysical applications(8). Voronoi-tessellated Markovian 117 milestoning is an implementation that allows reconstruction of the long-time dynamics of a system 118 from independent simulations confined within a set of cells spanning the space of the reaction 119 coordinates $(9)$. This method has successfully captured the rates of CO entry/exit in myoglobin(10), 120 and recently the ligand binding kinetics(11). Here, we make use of the "soft-walls" version, which 121 confines the sampling within the Voronoi cells using flat-bottom harmonic restraining 122 potentials(12). This approach is easy to implement, allowing us to take advantage of CUDA 123 accelerated MD packages, and has been shown to yield the same results as the original "hard-walls" 124 version, which instead inverts atomic velocity at the cell boundaries. The "soft-walls" version has 125 been used to study nucleation of an ionic liquid(13) and ion permeation across a claudin-15 126 paracellular channel(14). Here we adopt this approach to explore the permeation of cAMP through 127 a Cx26 hemichannel. 


\section{Results}

\section{CAMP permeation under opposite voltages}

131 We first investigated inward/outward cAMP permeation rates under voltages in the presence of 132 symmetric cAMP concentrations. To obtain a sufficient number of transition events and ensure 133 unidirectional flux, we applied $+/-200 \mathrm{mV}$ transmembrane potentials to accelerate permeation. 27 134 cAMP molecules and $27 \mathrm{Mg}^{2+}$ ions, corresponding to $26.5 \mathrm{mM}$, were present on each side of the 135 membrane. To mimic physiological salt concentration and also to neutralize the protein charge, 82 $136 \mathrm{~K}^{+}$and $163 \mathrm{Cl}^{-}$ions were added to the bulk aqueous compartments, corresponding to $80.5 \mathrm{mM} \mathrm{K}^{+}$ 137 and $160.1 \mathrm{mM} \mathrm{Cl}^{-}$(Table S1). Using $\mathrm{z}<|50| \AA$ as boundaries of the hemichannel (center-of-mass 138 of protein is at $\mathrm{z}=0 \AA$ ), 9 single-molecule transitions were observed at $+200 \mathrm{mV}$ and 12 transitions 139 at $-200 \mathrm{mV}$ during each $2 \mu$ s simulation (Figure 1, Table 1, and raw data in Table S2). At each 140 voltage, all transitions were in the same direction.

141 To estimate the uncertainty due to the small number of events, we calculated the confidence 142 interval for the average transition time $(<t>)$ of cAMP through the channel by fitting the transition 143 times to an exponential distribution. Based on a maximum likelihood estimation, MFPT is $448 \mathrm{~ns}$ 144 at $+200 \mathrm{mV}$ with $95 \%$ confidence interval (CI95) of 204-1646 ns, and 510 ns with CI95 of 249$1451571 \mathrm{~ns}$ at $-200 \mathrm{mV}$. The average time between consecutive transition events $(\tau=$ length of 146 simulation/number of transits) is $222 \mathrm{~ns}$ at $+200 \mathrm{mV}$ and $166 \mathrm{~ns}$ at $-200 \mathrm{mV}$. Given the confidence 147 intervals, there is no significant difference in the flux in each direction. The ratio $<t>/ \tau$ is 2.0 and 148 3.1, for $+200 \mathrm{mV}$ and $-200 \mathrm{mV}$, respectively, which indicates there are on average two to three 149 cAMP molecules in the channel at any given time at each voltage. Inspection of the trajectories 150 shows that this is only due to the accumulation of cAMP molecules at the intracellular entrance 151 (Figures 2 and 3).

152 Although the experimental transition time of cAMP under voltage is not available, a $153 \mathrm{cAMP} / \mathrm{K}^{+}$permeability ratio of 0.027 was reported using simultaneous measurements of $\mathrm{Cx} 26$ 154 junctional conductance and reporter-based intercellular transfer of cAMP(15). We calculated $\mathrm{K}^{+}$ 155 transition time in presence of $27 \mathrm{cAMP}$ is $13.1 \pm 13.0 \mathrm{~ns}$ at $+200 \mathrm{mV}$ and $6.8 \pm 7.8 \mathrm{~ns}$ at $-200 \mathrm{mV}$. 156 If we assume the time needed to cross the junctional channel (two hemichannels docked at the 
extracellular ends) is the sum of the transition time in two opposite directions, we obtain a $\mathrm{cAMP} / \mathrm{K}^{+}$ratio of 0.021 , reasonably close to the experimental ratio.

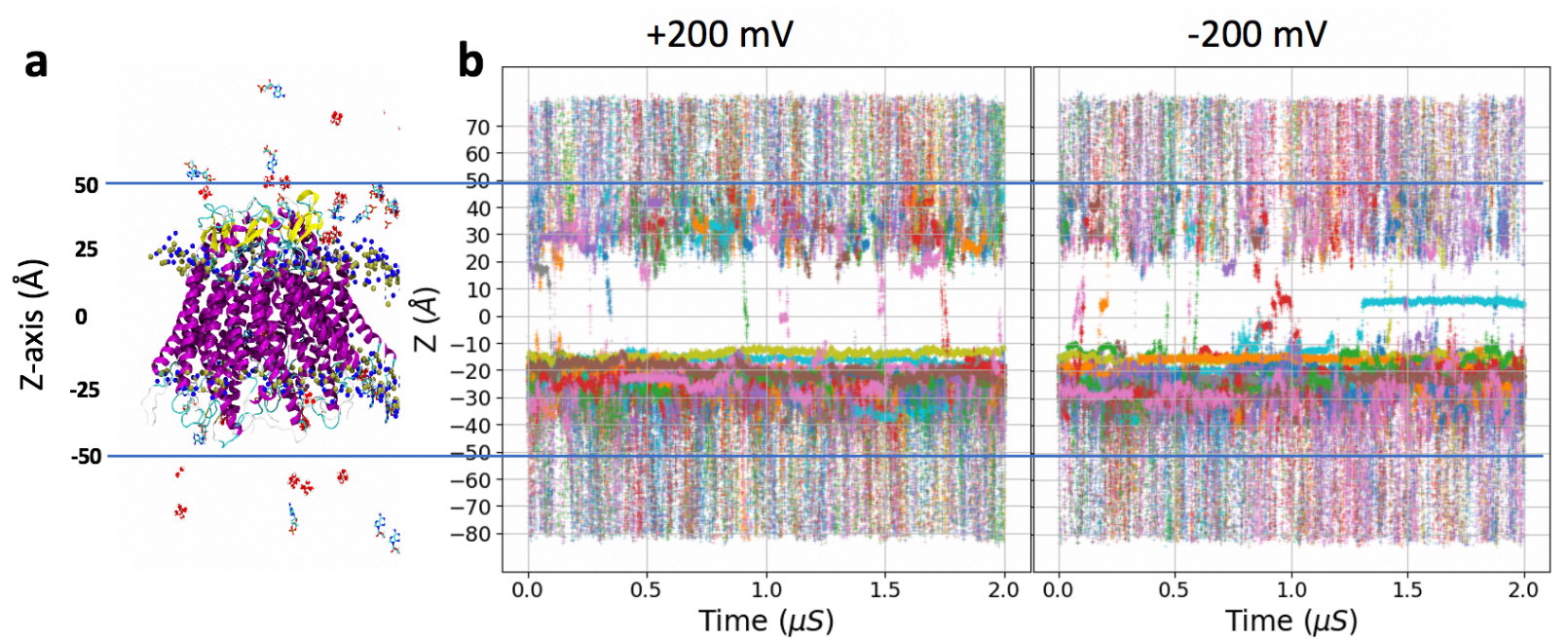

Figure 1. Simulations of cAMP permeation through $\mathrm{Cx} 26$ at $+200 \mathrm{mV}$ and $-200 \mathrm{mV}$ membrane potential. a. Snapshot of the simulated system. Protein backbone is shown in cartoon mode and colored by the secondary structure (helix in magenta, beta-sheet in yellow, disordered loop in cyan). cAMP molecules are shown in licorice with atoms colored (red oxygen, cyan carbon, blue nitrogen, yellow phosphate). Lipids, ions, and water molecules are not shown. b. The z-coordinates of all 27 cAMP molecules in the system during the simulations are shown in different colors. Note that the flux of cAMP is in the direction opposite to the field; thus, cAMP flux is inward (down in this figure) under $+200 \mathrm{mV}$ and outward under $-200 \mathrm{mV}$.

Table 1. Transition time of cAMP through $\mathrm{Cx} 26$ hemichannel under voltage.

\begin{tabular}{|c|c|c|}
\hline & $\mathbf{+ 2 0 0} \mathbf{m V}$ & $\mathbf{- 2 0 0} \mathbf{m V}$ \\
\hline cAMP flux direction & Inward & Outward \\
\hline Transition events in $2 \mu \mathrm{s}$ & 9 & 12 \\
\hline Events per $\mu \mathrm{s}$ & 4.5 & 6.0 \\
\hline Time between transition events & $222 \mathrm{~ns}$ & $166 \mathrm{~ns}$ \\
\hline Mean transition time $\left(\mathrm{CI}_{95}\right) *$ & $448 \mathrm{~ns}(204-1646)$ & $510 \mathrm{~ns}(249-1571)$ \\
\hline Mean dwell time $\left(\mathrm{CI}_{95}\right)$ & $305 \mathrm{~ns}(149-940)$ & $398 \mathrm{~ns}(205-1086)$ \\
\hline Mean barrier crossing time $\left(\mathrm{CI}_{95}\right)$ & $143 \mathrm{~ns}(79-330)$ & $106 \mathrm{~ns}(54-290)$ \\
\hline
\end{tabular}

*Sample mean and 95\% confidence intervals are based on Maximum Likelihood Estimate by fitting the analysis/blob/master/confidence_interval_exponential.ipynb. 


\section{Pore cAMP density profiles from voltage simulations}

179 The density profiles accumulated from voltage simulations can provide an estimate of the locations

180 of energetic barriers and binding sites. The charge densities of protein, lipids, total ions, and 181 individual ions $\left(\mathrm{K}^{+}, \mathrm{Cl}^{-}, \mathrm{cAMP}^{-}, \mathrm{Mg}^{2+}\right)$ are plotted along the channel z-axis (Figure 2a). It can be

182 seen that the channel is largely positive at intracellular entrance where the highest density of $\mathrm{Cl}^{-}$, 183 cAMP- ions are located. The accumulation of cAMP at the intracellular entrance can also be seen 184 clearly in Figure 1. A volumetric map showing the 3D density of cAMP inside the channel from 185 the $-200 \mathrm{mV}$ simulation is illustrated in Figure 2b. A smooth indicator of cAMP distribution is 186 shown using Boltzmann inversion $(-k T \ln (\rho))$ of the cAMP density; we will call these log-density 187 plots. Figure 2c shows the 2D cAMP log-density along the z-axis and the radial axis $R$. Figure 2d 188 shows the 1D cAMP log-density within the pore along z. Note that these density profiles are 189 acquired from nonequilibrium simulations under the influence of voltage. They indicate how the cAMP behaves under the influence of both external voltage and the intrinsic free energy, hence do not represent the equilibrium free energy profiles. The symmetric cAMP density in the bulk regions on each side of the channel is the result of the periodic boundary condition used for MD simulations.

The 1D log-density profiles (Figure 2d) have a broad minimum at the intracellular entrance of the channel and a major peak in the middle of the channel. The shapes of the global minimum region are essentially identical under both voltages. There is a second well in the $-200 \mathrm{mV}$ density that appears to bisect the central peak. The dwell times of cAMP (Table 1) at the broad minimum $(-50<\mathrm{z}<10 \AA)$ are quite large under both voltages: $305 \mathrm{~ns}$ at $+200 \mathrm{mV}$, and $398 \mathrm{~ns}$ at $-200 \mathrm{mV}$ (Table 1). In contrast, the rest of the channel region $(-10<\mathrm{z}<50 \AA)$, which contains the major barriers, was crossed by cAMP more rapidly: $143 \mathrm{~ns}$ for inward flux (at $+200 \mathrm{mV}$ ), and $106 \mathrm{~ns}$ for outward flux (at $-200 \mathrm{mV}$ ).

The broad binding well $(-50<\mathrm{Z}<-10)$ present at both voltages is located in the C-terminal 204 frequency analysis between cAMPs and protein sidechains indicates that cAMP bind to R99/104 205 and K103 on TM2 over 70\% of the simulated time, and bind to K102 and R98 on TM2, and K15 206 on NTH over 30\% of the time (Figure 3a), thus providing a basis for the cAMP accumulation at 207 this region. Further investigation revealed that the cAMP forms clusters between $-30<\mathrm{z}<0 \AA$, while 208 almost no clusters are found in the rest of the channel (Figure 3b). Most of the clusters contain 2 
209 cAMPs, which interact through pi-stacking of adenosine rings. Clusters of 3 cAMPs forming

210 hydrogen bonds with each other also exist (Figure 3c). These clusters are not seen in the bulk. It

211 appears that the positively charged residues at the intracellular entrance of the pore facilitate cAMP

212 clustering by reducing the translational and rotational entropy of the molecules. To check whether

213 this accumulation produce a "crowding" effect on entry into the pore, we compared the unoccupied

214 lumen radius with and without cAMP molecules present. Figure S1 suggests that the accumulation

215 of cAMP had little effect on the average available cross-sectional area at the pore entrance. In

216 addition, the peaks in the two log-density profiles (Figure 2d) do not closely correlate with the

217 narrow regions of the pore (see Figure S1 and Figure 8a for two different radius measurements),

218 indicating that steric hindrance is not a major contributor to the cAMP transition barrier. 


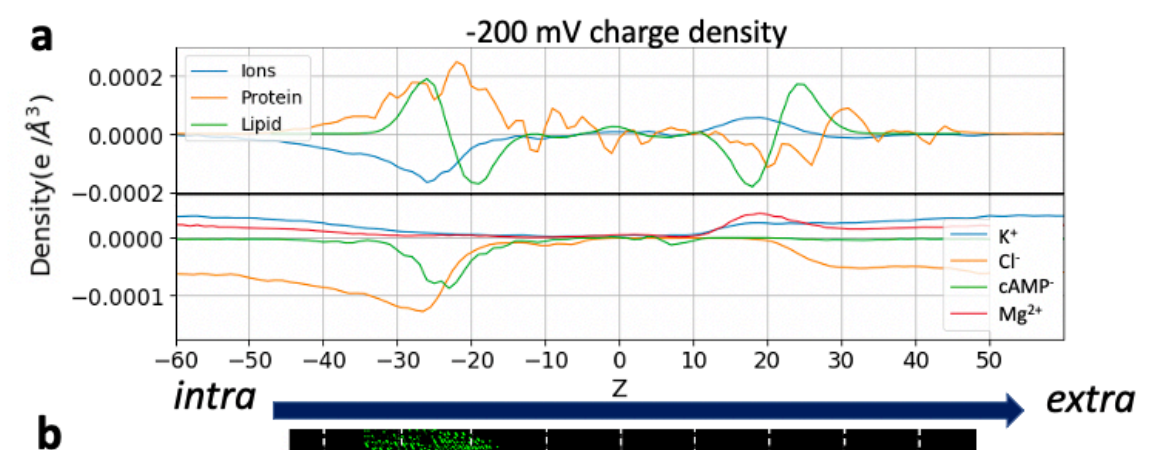

b

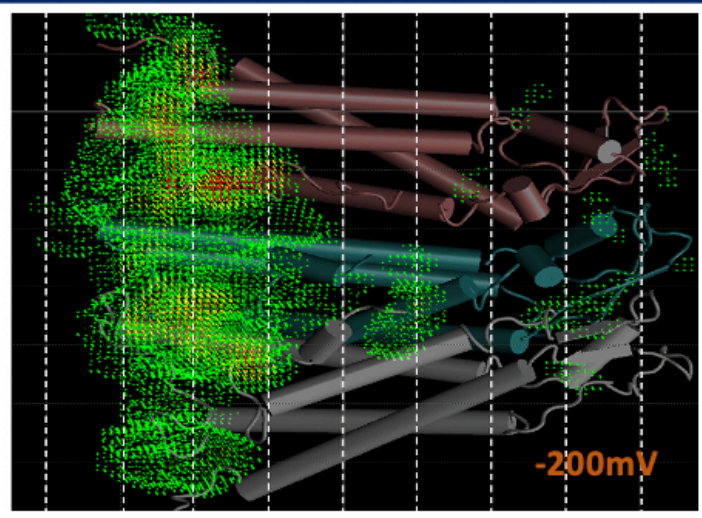

C
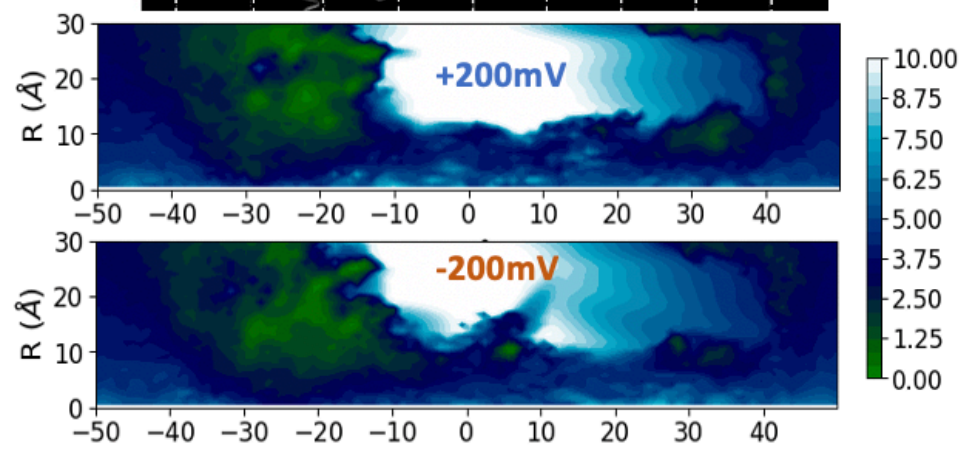

d

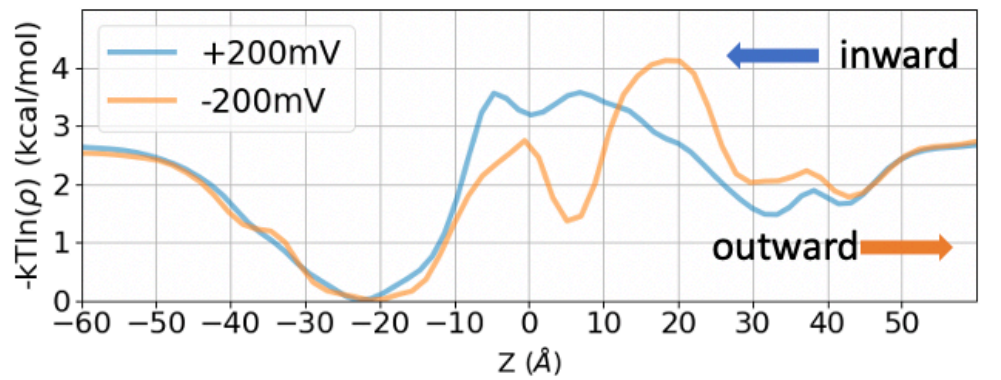

221 Figure 2. Charge density profile and cAMP log-density profiles in 3D, 2D, and 1D. a. Charge density profiles of protein, lipids, total ions and individual charges groups $\left(\mathrm{K}^{+}, \mathrm{Cl}^{-}, \mathrm{cAMP}^{-}, \mathrm{Mg}^{2+}\right)$ along the channel z-axis obtained from the $-200 \mathrm{mV}$ trajectory. b. Volumetric map of cAMP 3D density under $-200 \mathrm{mV}$ simulation. c. Boltzmann inversion $-k \operatorname{Tn}(p(z))$ of the accumulated cAMP density profile in $2 \mathrm{D}$ along the channel z-axis and radial axis R. d. 1D log-density plot of the cAMP density profile within the pore along the channel z-axis. Arrows indicate the direction of cAMP flux at each voltage. Note: a represents charge density in the entire simulation system, while $\mathbf{b}$, $\mathbf{c}$, and $\mathbf{d}$ depicts the cAMP density only within the pore using the same cylindrical radius cutoff of $30 \AA$ as in the milestoning simulation below. 
a

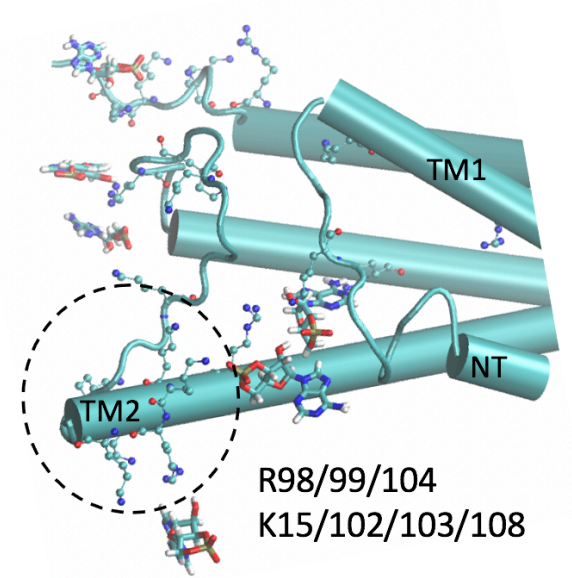

b

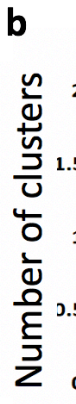

231

232

233

234

235

236

237

238

239

240

241

242

243

244

245

246

247 c
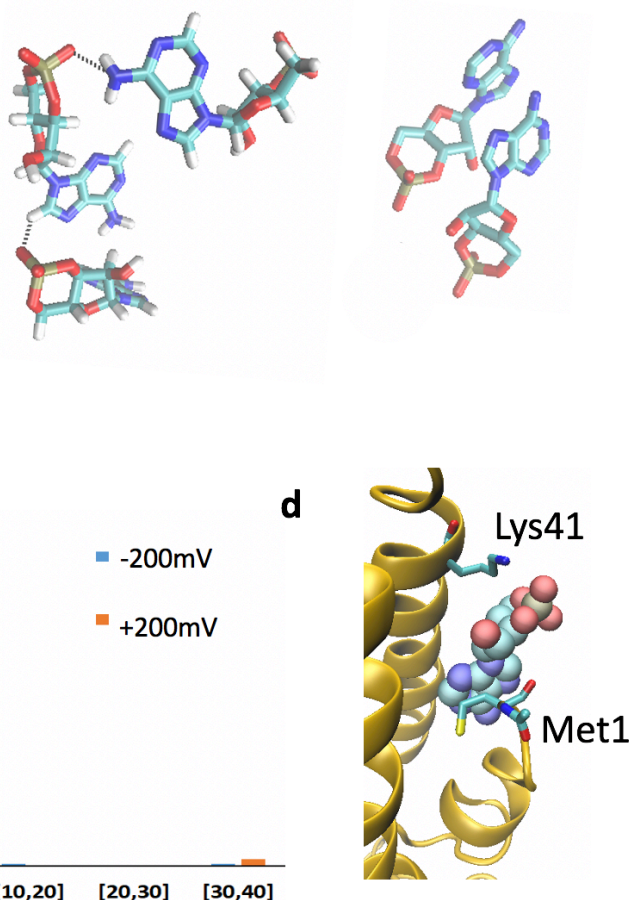

Figure 3. Permeant-permeant interactions and permeant-channel interactions. a. Snapshot showing cAMP molecules (in licorice) near positively charged residues (in CPK mode) at the intracellular entrance. Protein is in cartoon mode. b. Number of cAMP clusters in each $10 \AA$ window during $+/-200 \mathrm{mV}$ simulations. A cluster is defined as at least two cAMP molecules with centers of mass within $20 \AA$ at any time during the simulations. c. Snapshots of two cAMPs clustered via pi-stacking and three cAMPs clustered via hydrogen bonds. d. Snapshot showing one cAMP trapped between Lys41 and Met1.

During one of the 12 permeation events at $-200 \mathrm{mV}$ simulation, one cAMP was trapped between Met1 in the N-terminal helix (NTH) and Lys41 on the first transmembrane helix (TM1) (Figure 1b, 3d). This was reflected in the density profile as a large dip in the peak at $\mathrm{z}=5 \AA$ (Figure 2d). Both NTH and Lys41 have been suggested to be involved in voltage-sensing in Cx26(16, 17). This raised the question of whether the instance of a long residency of a cAMP molecule at this particular position at $-200 \mathrm{mV}$, but not evident at $+200 \mathrm{mV}$ or $0 \mathrm{mV}$ (from PMF milestoning below) is a consequence of voltage-driven repositioning of these charged moieties. To evaluate their responses to the local electric field, we plotted the angles between the z-axis and the principal 
vector of Lys41 or NTH (residues 1 to 11 ) during $2 \mu$ s simulations at $+/-200 \mathrm{mV}$ (Figures S2a and S2b). Except for a clear reduction in Lys41 fluctuation in subunit 5, where the cAMP was trapped, there is no clear preference in the orientation of the Lys41 or NTH in response to the two opposite voltages. Therefore, this trapped cAMP is unlikely to be due to the effect of voltage on

252 the protein. Of course, this result does not indicate that the NTH and Lys41 are uninvolved in voltage-sensing, only that they did not respond to $+/-200 \mathrm{mV}$ within the $2 \mu$ s simulations.

\section{cAMP permeation free energy using Markovian milestoning}

256 The simulations above provide a nonequilibrium view of the cAMP transition process driven by voltage. Two opposite voltages resulted in similar cAMP density profiles and similar ranges of

258 flux rates at each voltage. However, the small number of stochastic events (21 in total) led to the

259 large uncertainty in the mean passage time of the transition event. In contrast to a long trajectory

260 exploring the whole channel, multiple MD simulations confined in intervals partitioning the space can offer sufficient statistics within a shorter running time. Here, we used Voronoi-tessellated Markovian milestoning MD simulations (hereafter referred to as milestoning simulation) on a tessellation along the $\mathrm{z}$-coordinate of the center-of-mass of a single cAMP to estimate the PMF and the kinetics of permeation through the channel at zero membrane voltage.

Figure 4 shows the 1D PMF (in red) obtained from the equilibrium probability of finding cAMP in each milestoning cell. This PMF of a single cAMP permeating without applied voltage has features similar to the log-density plots of the accumulated density of cAMP from the voltage simulations. A free energy barrier spans between $-20<\mathrm{z}<30 \AA$, with the peak located at $\mathrm{z}=+20 \AA$, the same as the peak in log-density from the $-200 \mathrm{mV}$ simulation. The height of the barrier relative to bulk $(1.2 \mathrm{kcal} / \mathrm{mol})$ is slightly lower than in $-200 \mathrm{mV}$ log-density $(1.8 \mathrm{kcal} / \mathrm{mol})$ and higher than in $+200 \mathrm{mV}(0.8 \mathrm{kcal} / \mathrm{mol})$. The major well on around $\mathrm{z}=-20 \AA$ from voltage simulations are

272 much broader and more favorable $(-2.8 \mathrm{kcal} / \mathrm{mol}$ relative to bulk) than the well in the PMF $(-2$ $273 \mathrm{kcal} / \mathrm{mol}$ ), likely due to the cAMP clustering, which is absent in the single cAMP milestoning. The 274 dip that split the peak at $\mathrm{z}=+5 \AA$ under $-200 \mathrm{mV}$ also shows up in the PMF, but with much smaller 275 magnitude. It is thus possible that the magnitude of the dip at $-200 \mathrm{mV}$ in the log-density plot is 276 overestimated due to the contribution of the single, rare event of cAMP trapping (evident in Figure 277 1b). 
Similar to the voltage simulations, milestoning simulation without voltage reveals two

279 binding sites for cAMP, a major one (site 1) near the intracellular entrance between NT and TM2

280 (-2 kcal/mol relative to bulk value) and a (bisected) much smaller one (site 2$)$ at the extracellular

281 loop (E1) region $\left(-0.7 \mathrm{kcal} / \mathrm{mol}\right.$ relative to bulk). The single cAMP dissociation constant, $K_{D}$, can

282 be estimated from the single cAMP equilibrium PMF using Eq. 1:

$$
1 / K_{D}=\pi R^{2} \int_{z_{\min }}^{z_{\max }} d z e^{-W(z) / k_{B} T}
$$

Eq. 1

$284 W(\mathrm{z})$ is the PMF with bulk as reference. $R$ is the radius of a cylindrical restraint (30 $\AA$ ). Integrals 285 over individual energy wells indicate that cAMP will bind to the intracellular site (binding site 1: $286-43.4<\mathrm{z}<18.6 \AA$ ) with $K_{D}$ of $1.8 \mathrm{mM}$ and to the extracellular site (binding site $2: 18.6<\mathrm{z}<60.6 \AA$ ) 287 with $K_{D}$ of $7.3 \mathrm{mM}$ (Figure 4a). The integral over the entire PMF yields a total $K_{D}$ of $1.4 \mathrm{mM}$ for 288 the channel. Thus, at the bulk cAMP concentration of the voltage simulations $(26.5 \mathrm{mM})$, both 289 binding sites are likely highly occupied, while at the concentration equivalent to the single cAMP 290 used in milestoning $(1 \mathrm{mM})$, none of the binding sites would be occupied more than $50 \%$. 


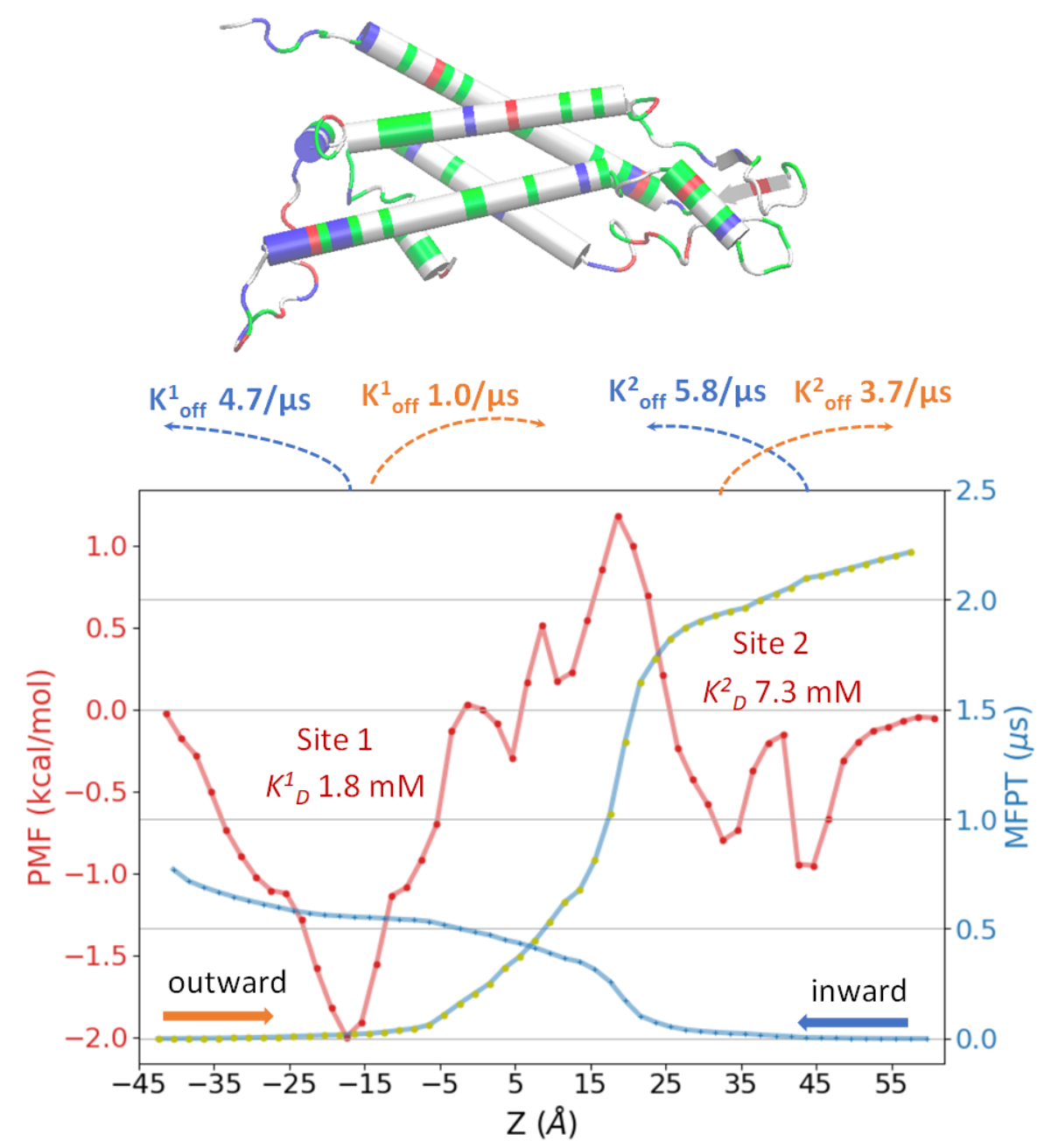

Figure 4. Free energy and kinetics of a single cAMP permeation through $\mathrm{Cx} 26$ hemichannel from milestoning simulation $(\mathbf{V}=\mathbf{0} \mathbf{~ m V})$. Potential of mean force (PMF) in red, inward mean first passage times (MFPT) in blue, and outward MFPT in yellow dotted blue line. Binding constants and dissociation rates derived from PMF and MFPT profiles are indicated. The graphic at the top shows the backbone of one Cx26 subunit with z-positions aligned with the plot below (basic residues in blue, acidic in red, polar in green, and nonpolar in white).

\section{cAMP permeation kinetics using Markovian milestoning}

According to the MFPT profiles in Figure 4, from the first to the last milestone at the boundaries of the channel, it takes about $0.77 \mu$ s for inward flux and $2.22 \mu$ s for outward flux.

303 Thus, the inward flux is 2.9 times faster than outward flux once a single cAMP enters the channel.

304 The faster inward than outward flux is the direct consequence of the asymmetric channel, reflected 305 in the asymmetric PMF showing a maximum of $3.2 \mathrm{kcal} / \mathrm{mol}$ barrier for outward flux, which takes 
$1.0 \mu$ s to cross, while the two smaller barriers of $2.0 \mathrm{kcal} / \mathrm{mol}$ for inward flux only take $212 \mathrm{~ns}$ (site 1) and $145 \mathrm{~ns}$ (site 2) to cross. MFPT profiles can provide direct information about the dissociation rate $\left(\mathrm{k}_{\mathrm{off}}\right)$ of a single cAMP. For outward permeation, $\mathrm{k}_{\mathrm{off}}{ }^{1}$ for binding site 1 is $1.0 \mu \mathrm{s}^{-1}(-17.4$ $<\mathrm{z}<18.6)$ and $\mathrm{k}_{\text {off }}{ }^{2}$ for binding site 2 is $3.7 \mu^{-1}(32.6<\mathrm{z}<60.6)$. For inward permeation, $\mathrm{k}_{\text {off }}{ }^{1}$ is 4.7 $\mu \mathrm{s}^{-1}(-41.4<\mathrm{z}<-17.4)$ and $\mathrm{k}_{\mathrm{off}}^{2}$ is $3.7 \mu \mathrm{s}^{-1}(18.6<\mathrm{z}<42.6)$. With the equilibrium constant $K_{D}$ from PMF, we can also estimate the association rate $\mathrm{k}_{\text {on }}{ }^{1}$ of $0.6 \mu \mathrm{s}^{-1} \mathrm{mM}^{-1}$ and $\mathrm{k}_{\text {on }}{ }^{2}$ of $0.5 \mu \mathrm{s}^{-1} \mathrm{mM}^{-1}$ for outward permeation, and $\mathrm{k}_{\text {on }}{ }^{1}$ of $2.6 \mu \mathrm{s}^{-1} \mathrm{mM}^{-1}$ and $\mathrm{k}_{\mathrm{on}}{ }^{2}$ of $0.8 \mu \mathrm{s}^{-1} \mathrm{mM}^{-1}$ for inward permeation.

It should be noted that the MFPT is subjected to the condition that the channel is occupied by only one permeating molecule at any time. Thus, it only represents the transit time of a single permeant traversing an otherwise permeant-free channel. The full kinetics and flux at finite bulk concentrations of the permeant also depend on bulk diffusivity and concentration, and the diameters of the entrance at each end of the pore. For instance, diffusion current to a disk-like adsorber is $\boldsymbol{I}=\mathbf{4 D} \boldsymbol{R} \boldsymbol{C}$, where $\boldsymbol{C}$ is the permeant concentration in the infinite bulk, $\boldsymbol{D}$ is bulk diffusion constant, and $\boldsymbol{R}$ is the radius of the disk-shaped absorber(18). Interestingly, for Cx26 hemichannel, the intracellular entrance (radius $\boldsymbol{R}=25 \AA$ ) is larger than the extracellular entrance $(\boldsymbol{R}=10 \AA)$ (see pore radius in Figure 8a). Taking into account the effective radius of cAMP as $r=3 \AA$ (calculated from radius of gyration), the effective pore radii are $22 \AA$ and $7 \AA$ for the intracellular and extracellular pore entrances, respectively, indicating that it is $\sim 3$ fold more likely for cAMP to reach the pore by random diffusion from bulk to the intracellular than the extracellular side.

Quantitative experimental measurements of the flux of cAMP through Cx26 channels in cells are very complex and subject to a variety of potential confounding factors (5). Two studies, which used different indirect strategies to report cAMP flux through junctional channels in the absence of junctional voltage, yielded estimates of cAMP permeability that differed by nearly a factor of $8\left(6.2\right.$ and $\left.47 \times 10^{-3} \mathrm{um}^{3} \mathrm{sec}^{-1}\right)(15,19)$. Using the volume of the Cx26 channel obtained

330 from the simulations, these permeability predict cAMP transit rates through junctional channels of

$33114 \mu \mathrm{s}$ and $1.9 \mu \mathrm{s}$, respectively, which bracket the $\sim 3 \mu \mathrm{s}$ transit time inferred from our studies (sum of hemichannel "outward" and "inward" transit times of 0.77 and $2.22 \mu \mathrm{s}$ ). 


\section{Influence of voltages on free energy profile}

336 The milestoning simulations at zero voltage yielded an inward transition time is 2.9 times faster 337 than the outward transition time ( 0.77 vs $2.22 \mu \mathrm{s})$. Interestingly, the mean inward/outward barrier 338 crossing time under voltages are quite similar: $143 \mathrm{~ns}\left(\mathrm{CI}_{95}\right.$ 79-330 ns) at $+200 \mathrm{mV}$, and $106 \mathrm{~ns}$ 339 (CI95 54-290 ns) at $-200 \mathrm{mV}$ (Table 1). Below we show that this is likely due to the negative 340 voltage reducing the free energy barrier for cAMP outward flux.

It has been shown previously that the total PMF under voltage $W_{\text {tot }}(z)$ can be computed

343 PMF derived from milestoning), and the additional potential introduced by the external field $344 q \delta \phi(z)$ (Eq. 2)(20-22). This additional potential has two components. One is the constant electric 345 field throughout the entire simulated periodic cell $E=V(z) / L_{Z}$, where $V(z)$ is the voltage linear to $346 L_{Z}$, the length of the PBC box in the $z$-direction. The other component is the reaction potential due 347 to the voltage-induced changes of the spatial distribution and orientation of the water dipole and 348 mobile ions, as well as flexible and charged atoms in the protein and membrane(23). This approach 349 presumes that the channel and the permeant do not undergo substantial conformational changes 350 due to the external field within the time of simulation ( $2 \mu \mathrm{s}$ in this case). This assumption is 351 supported by our results from $+/-200 \mathrm{mV}$ simulations showing highly similar cAMP density 352 distribution (Figure 2) and pore radius profile (Figure S1).

$$
W_{t o t}(z)=W_{e q}(z)+q \delta \phi(z)=W_{e q}(z)+q\left(V(z)+\phi_{v}(z)-\phi_{0}(z)\right)
$$

The reaction potential introduced by external field may be approximated by the difference in electrostatic potential in presence and absence of the external potential, $\phi_{v}(z)-\phi_{0}(z)$. To calculate this difference in electrostatic potential, three additional $100 \mathrm{~ns}$ simulations at $-200,0$, $+200 \mathrm{mV}$ were carried out for the same system but without cAMP. 3D electrostatic potential maps $\phi(\mathrm{r})$ were calculated based on all charged atoms in the simulated system $\rho_{i}(r)$ by solving the Poisson equation $\nabla^{2} \phi(r)=-4 \pi \sum_{i} \rho_{i}(r)$ on a 1 Å resolution grid using the VMD PMEPot plugin(24).

362 Figure 5a and $\mathbf{5 b}$ show the 1D potential along the central pore $\mathrm{z}$-axis without voltage (in blue) and under $+/-200 \mathrm{mV}$ (in orange). 

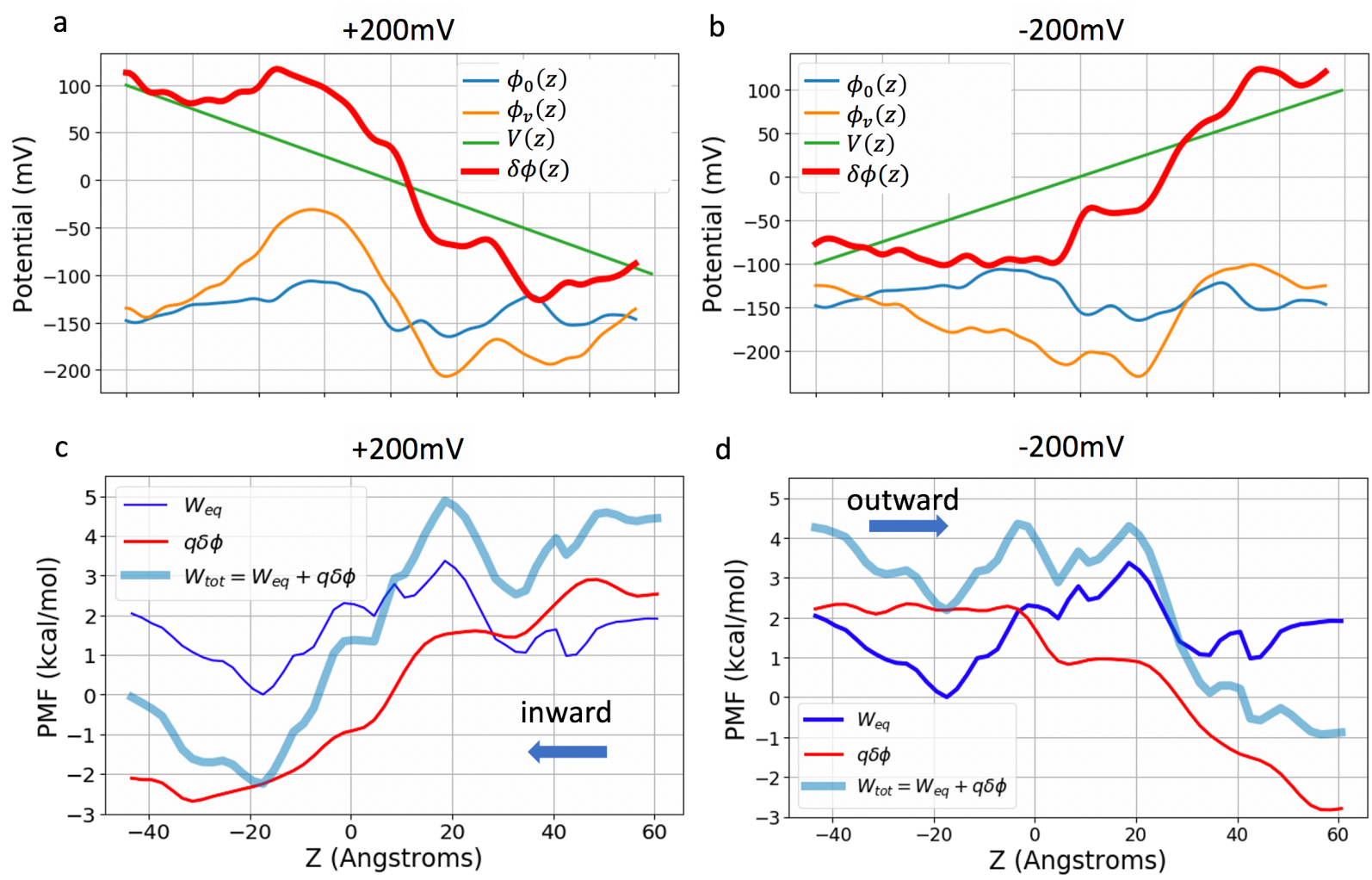

Figure 5. Electrostatic potentials and PMFs under voltages. a,b: applied potential in green $(+/-200 \mathrm{mV})$ and the electrostatic potential under voltage $\phi_{v}(\mathrm{z})$ in orange, and without voltage $\phi_{0}(\mathrm{z})$ in blue. The sum of applied potential and reaction potential (see Eq. 2) in shown in red. c,d: Total PMF under a constant electric field, $\mathrm{W}_{\text {tot }}(\mathrm{z})$, from the intrinsic PMF in absence of an electric field $\mathrm{W}_{\mathrm{eq}}(\mathrm{z})$, and the additional electrostatic potential energy introduced by the applied field $\mathrm{q} \delta \phi(\mathrm{z})$.

Cx26 is largely positive in the intracellular side $(-40<z<0)$, negative near the extracellular side $(15<\mathrm{z}<25)$, and slightly positive near extracellular entrance $(25<\mathrm{z}<35)$ (Figure 2a), thus it has an overall dipole vector pointing towards the positive z-direction. Consequently, within the protein-membrane region, positive voltage produces a potential $\phi_{v}(z)$ that enhances the protein dipole, while the one from negative voltage counters the protein dipole (Figure 5ab orange lines). In the bulk region and lipid headgroup region $(\mathrm{z}>|15|)$, the $\phi_{v}(z)$ nearly cancels out the external field $V(z)$. The sharp drop of the $\phi_{v}(z)$ under $+200 \mathrm{mV}$ can be visualized on 2D-electrostatic potential maps of the whole system (Figure 6a), and the 3D-electrostatic potential overlaid onto the solvent mass density iso-surface (Figure 6b) or onto the cAMP density iso-surface (Figure 6c). 
Figure 5 cd show the total PMF under each voltage $W_{\text {tot }}(z)$ obtained from Eq. 2. Clearly, $+200 \mathrm{mV}$ facilitates the inward flux of the negatively charged cAMP by increasing the free energy on the extracellular side of the protein $(\mathrm{z}>0)$ and decreasing the free energy on the intracellular side $(\mathrm{z}<0)$. The two inward flux energy barriers remain similar to those of the intrinsic PMF $(\sim 2.3$

$388 \mathrm{kcal} / \mathrm{mol})$. However, the outward $W_{\text {tot }}(z)$ at $-200 \mathrm{mV}$ significantly reduced the outward barrier 389 from $3.2 \mathrm{kcal} / \mathrm{mol}$ of the intrinsic PMF to $2.3 \mathrm{kcal} / \mathrm{mol}$. Thus, the asymmetry of the inward $v s$. 390 outward MFPTs of cAMP within the pore at zero voltage is eliminated by the external voltage. 391 This "voltage-equalizing" effect of permeation kinetics is a unique feature of large-pore channels 392 that have a protein dipole and mobile electrolytes inside the pore.
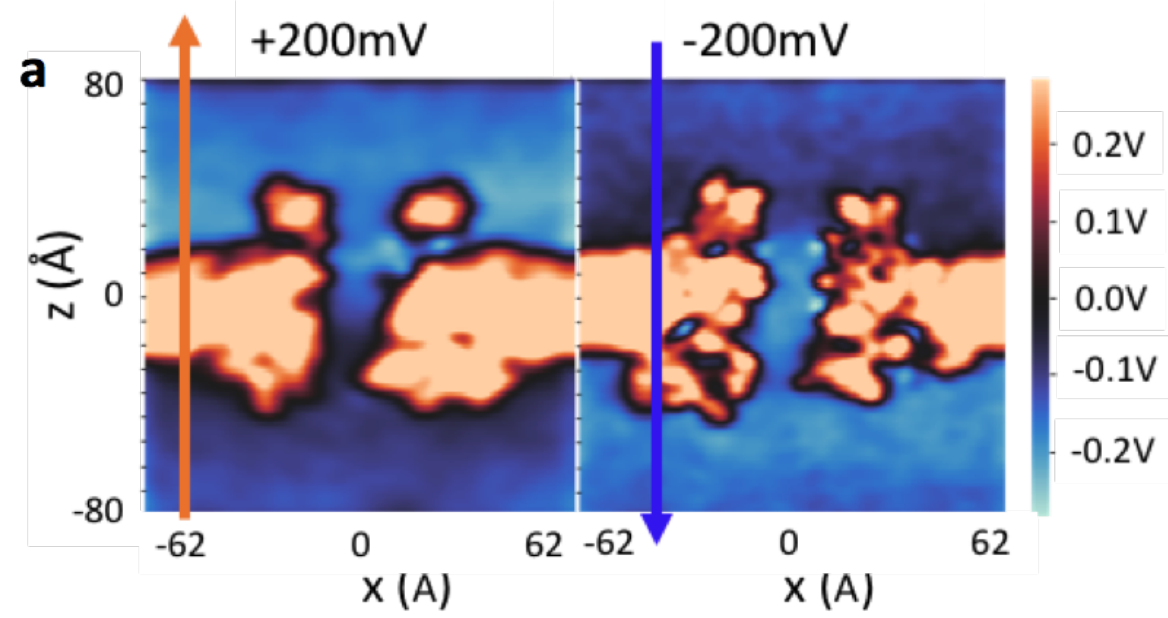

\section{b}
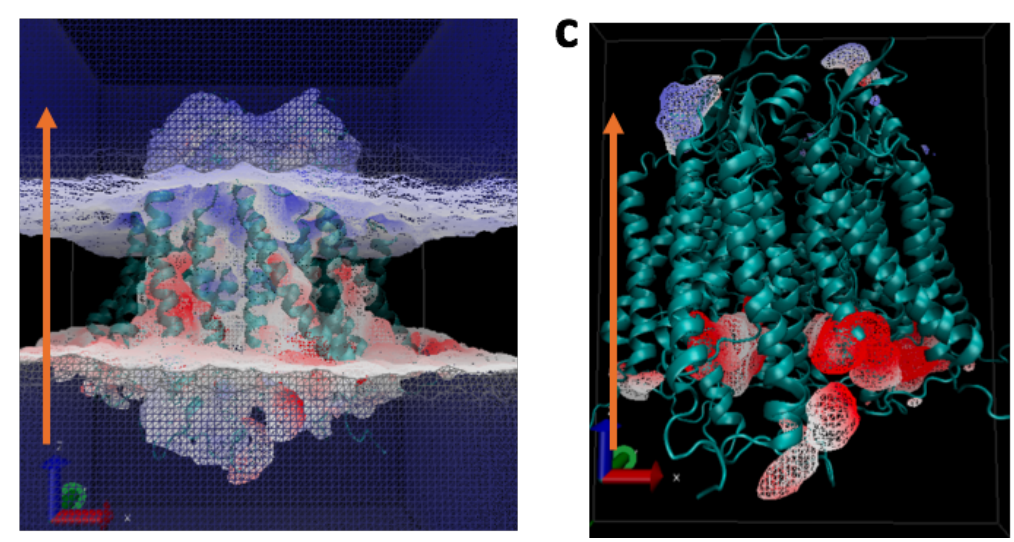

Figure 6. Electrostatic potential at $+200 \mathrm{mV}$ and $-\mathbf{2 0 0} \mathrm{mV}$ membrane potential. a. 2D-electrostatic potential maps (vector 1,0,1 in Cartesian space) at $+/-200 \mathrm{mV}$ voltage. b. The 3D-electrostatic potential at $+200 \mathrm{mV}$ overlaid onto the solvent mass density iso-surface. The color scale is -12.11 to $32.95 \mathrm{kT} / \mathrm{e}(-323$ 
in $-200 \mathrm{mV}$ is much less prominent (due to the opposite orientation of the intrinsic dipole as described in the text). c. Electrostatic potential overlaid onto the cAMP density iso-surface at $+200 \mathrm{mV}$. The color scale is -267 to $+267 \mathrm{mV}$. The iso-surface contour cutoff is $0.1 \mathrm{amu} / \AA^{3}$ for panels b and c. All data in this figure are calculated from the $3 \mathrm{D}$ electrostatic potential map $\phi(r)$ based on all charged atoms in the simulated system by solving Poisson's equation on a $1 \AA$ resolution grid using the VMD PMEPot plugin. PMEPot approximate point charge by a spherical Gaussian with an Ewald factor of $0.25 . \phi(r)$ is reported as the average of 1000 snapshots from the last $200 \mathrm{~ns}$.

\section{cAMP dipole orientations during transit with and without voltage}

cAMP is a fairly rigid molecule with a dipole moment of 37.1 Debye. Figure 7a shows the probability distribution of the angle between the cAMP dipole vector and the z-axis from milestoning simulations. Strikingly, the cAMP molecule rotates nearly 180 degrees five times on its way through the pore $(-40 \sim-20,-20 \sim 0,0 \sim 10,10 \sim 20$, and $20 \sim 40 \AA$ along the z-axis, also see Video1). To understand this, the z-components of the force vector acting on the cAMP from the rest of the system (protein, water, ions, lipids) are decomposed into electrostatics and vdW terms and plotted for each milestoning cell (Figure 7b). The reciprocal forces of electrostatics and vdW terms along the channel indicate that cAMP has close interaction with the walls of the pore lumen. The positive forces push cAMP in the positive z-direction, thus facilitating outward permeation, while the negative force facilitates inward permeation towards the negative $\mathrm{z}$-direction. The cAMP dipole rotates as the electrostatic force vector switches the sign. Thus, these re-orientations of 422 lumen.

What is the cAMP dipole orientation during the voltage induced transition? Figure 7c shows scatter plots of dipole vectors of permeating cAMP molecules from the two voltage simulations. Between the intracellular entrance and the major binding site (-40 -15 $\AA$ ), dipole angles show orientation under both voltages similar to those at zero voltage, likely due to the large magnitude of the channel local field. While the sampling is scarce in the barrier crossing region ($20<\mathrm{z}<20 \AA$ ), cAMP shows a clear preference in orientation at $\mathrm{z} \sim 0$ and at $\mathrm{z} \sim 25-30 \AA$, adopting the opposite orientation under the two voltages. Thus, this position-dependent dipole orientation of 
a

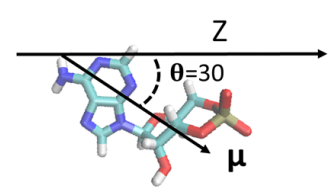

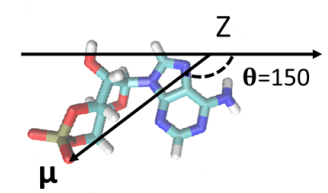

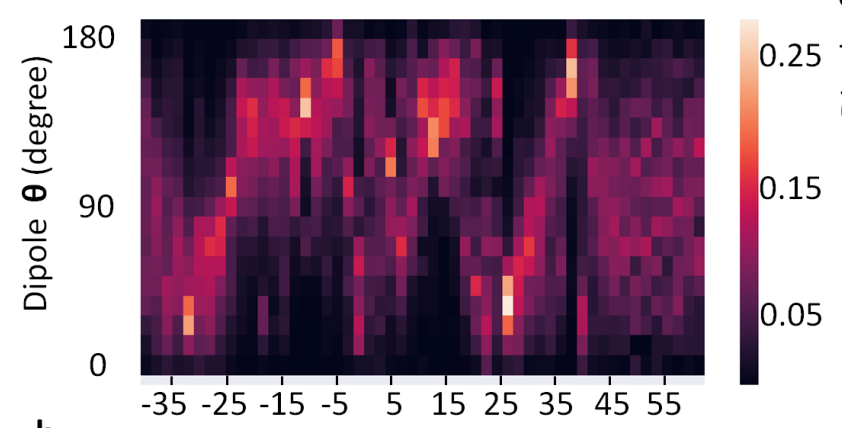

b

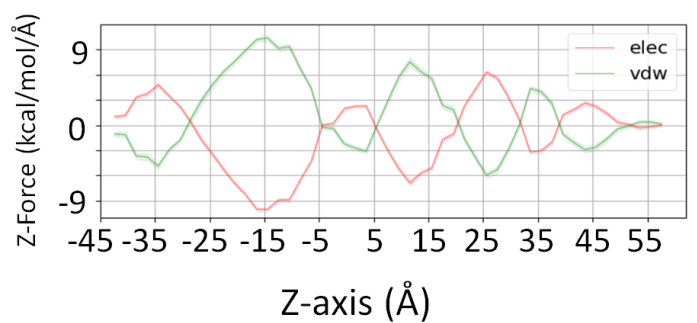

C
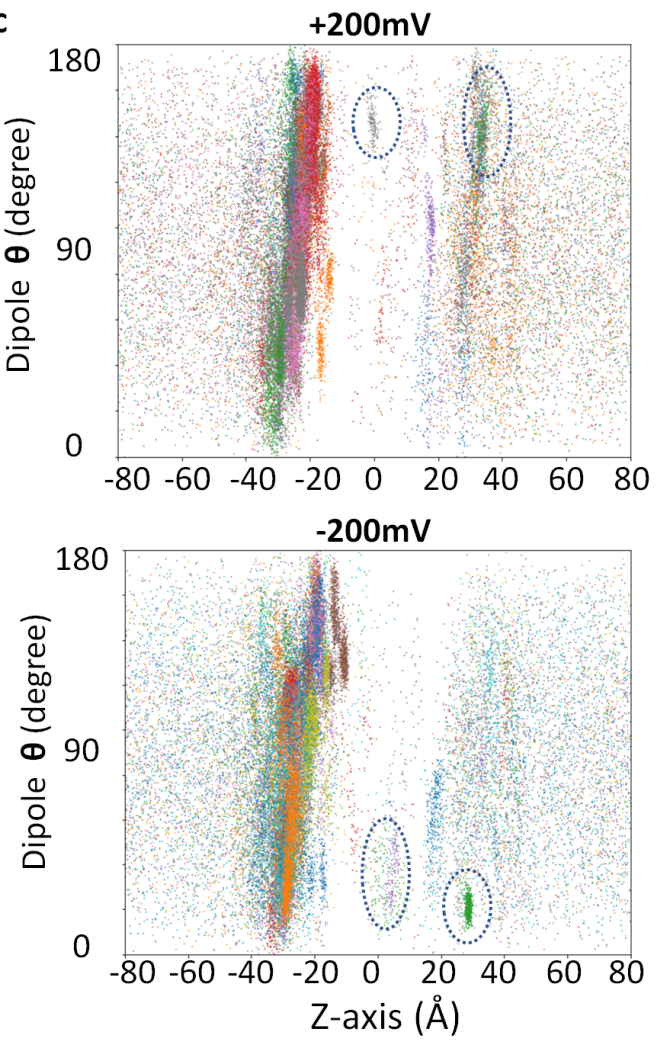

433 Figure 7. Dipole moment distribution and force decomposition for cAMP molecules along z-axis ( $\AA$ ). a. cAMP dipole angles are shown as a probability distribution for each milestoning simulation cell along the z-axis. Color bar shows the probability scale, lighter color represents higher probability. Representative dipole angles are illustrated on the cAMP molecule above. b. Mean electrostatic and vdW forces along zaxis on cAMP. Lines represent the running average of three milestoning cells and shaded areas represent standard error of mean within each milestoning cell. c. Dipole angle scatterplot of permeating cAMP at $200 \mathrm{mV}$ and $+200 \mathrm{mV}$ voltage simulations, respectively.

\section{Position-dependent influence of cAMP on pore radius}

The presence of cAMP at different locations inside the pore may have local or non-local

444 effect of the pore radius. To investigate the local effect, we first compared the mean and standard

445 deviations of the pore radius along the length of the pore, without cAMP (Figure 8a black), and

446 the radius at the position of the cAMP (Figure 8a blue). Notice that these pore radii, obtained

447 using grid-based cavity search program trj_cavity(25) ranged between 10 to $15 \AA$, larger than the

4487.5 to $10.5 \AA$ radius obtained using the Hole program (Figure S1). This is because the Hole

449 algorithm uses a spherical probe of increasing radius, which underestimates the space within an 
450 irregularly shaped pore. It is clear that the influence on pore radius depends where cAMP resides.

451 For instance, the radius increases when cAMP is around $\mathrm{z}=-10 \AA$ but decreases when it is around

$452 \quad z=30 \AA$, near the extracellular entrance of the pore.

453 To investigate the non-local effect of cAMP on pore radius, we used Cohen's d-score of 454 pore radius distributions to compare the mean radius with and without cAMP. Figure 8b is a heat 455 map of Cohen's d-score, which depicts the changes in pore diameter along its length (x-axis of 456 Figure $8 \mathrm{~b}$ ) caused by the presence of cAMP at each z-position (y-axis of Figure 8b). Cohen's d 457 score was calculated using Eq. 3:

$$
\text { Cohen's } d(z)=\frac{\left\langle r^{\prime}(z)\right\rangle-\langle r(z)\rangle}{S D_{\text {pooled }}}
$$

460 where $\langle r(z)\rangle$ is the average pore radius without cAMP (Figure 8a black) and $\left\langle r^{\prime}(z)\right\rangle$ is the 461 average pore radius with cAMP at various locations of the pore, obtained from milestoning 462 simulation trajectories. $S D_{\text {pooled }}$ is the pooled standard deviation of $r^{\prime}(z)$ and $r(z)$. For instance, 463 a d score of 6 (blue) means the presence of cAMP increased the mean pore radius by $6 S D_{\text {pooled }}$, 464 and a score of -6 (green) means the presence of cAMP decreased the mean pore radius by $4656 S D_{\text {pooled }}$.

466 On the heatmap, the $\mathrm{d}$ score colors along the diagonal line represent changes in pore radius 467 at the position of the cAMP, corresponding to the Figure 8a blue profile. The off-diagonal colors 468 represent non-local changes in pore radius away from where the cAMP is in the pore. It is evident 469 that the presence of cAMP can have effects on lumen radius distant from the position of cAMP 470 itself. Most interestingly, the scores on the upper off diagonal are more populated by green color 471 and lower off diagonal shows more blue color. This trend suggests that when cAMP permeates 472 through the pore, it tends to enlarge the radius on the left side (towards intracellular) and narrow 473 the radius on the right side (towards extracellular). 


\section{a}
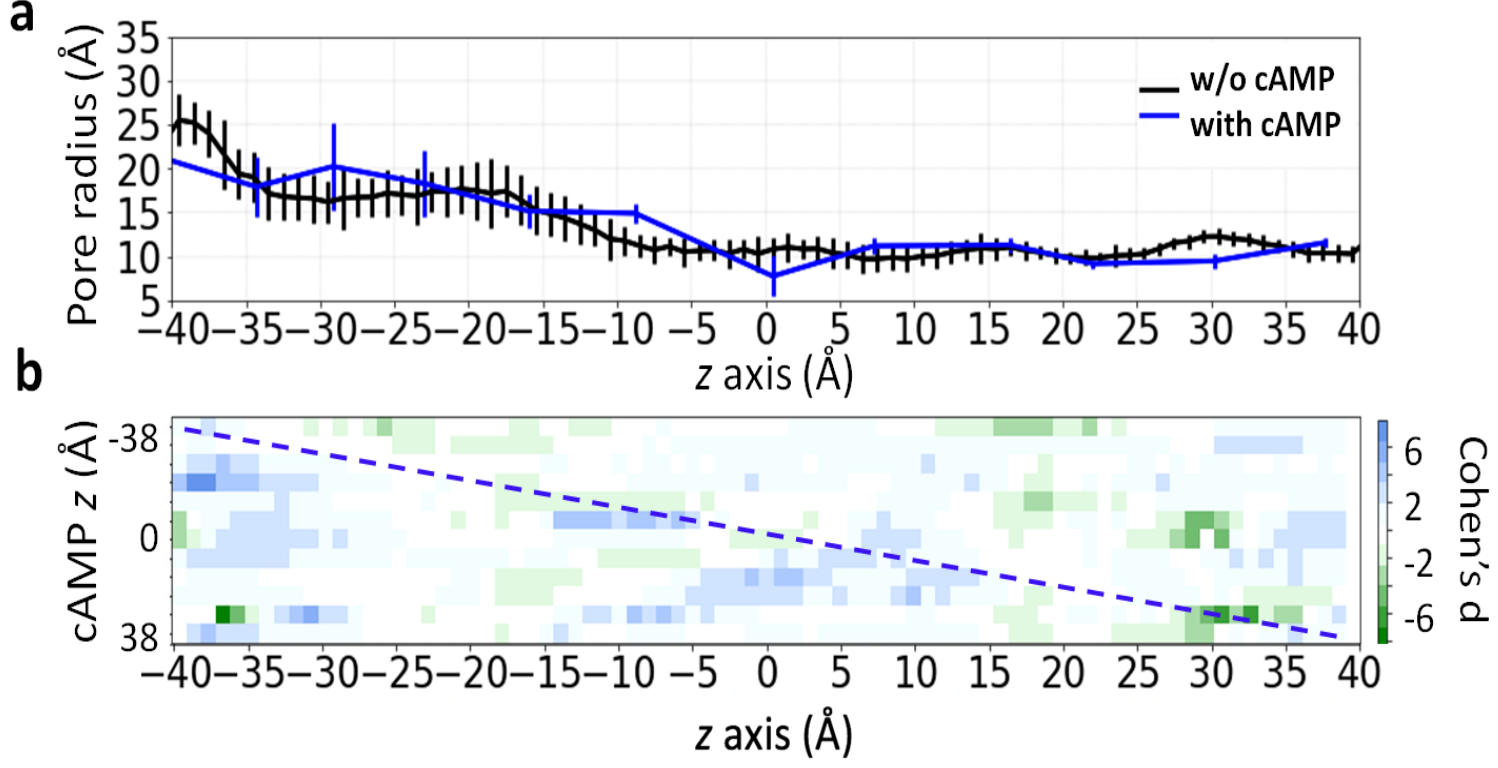

Figure 8. Local and non-local effect of cAMP on $\mathbf{C x 2 6}$ pore radius. a. The mean and standard deviation of $\mathrm{Cx} 26$ pore radius as a function of the pore axis (z) during simulations. Black line is the pore radius without cAMP and the blue line is the radius at the position of the cAMP. b. Cohen's d-scores of pore radius distributions (see Eq. 3). The y-axis indicates the z-position of the cAMP, and $\mathrm{x}$-axis is the fulllength pore axis. The dashed diagonal line indicates changes in pore radius at the position of the cAMP, while off-diagonal colors indicate cAMP's non-local effect on the pore radius. Blue color indicates cAMP increase the pore radius, and green color indicates cAMP decrease the pore radius.

\section{Discussion}

In this work, we use both long-timescale MD simulations and Voronoi-tessellated Markovian milestoning, an enhanced sampling method, to explore how a charged biological signaling molecule, cAMP, permeates a connexin pore. This work builds on our previously developed Cx 26 hemichannel model that was validated regarding ionic and molecular permeation properties $(6,26)$. We first obtained the density profile and the inward/outward cAMP flux under $+/-200 \mathrm{mV}$ voltages from two $2 \mu \mathrm{s}$ MD simulations in the presence of multiple cAMPs. These results were compared with the intrinsic potential of mean force (PMF) and the inward/outward mean first passage time (MFPT) of a single cAMP at zero voltage obtained from a total $16.5 \mu$ s of multi-replica milestoning. Those two computational approaches - long timescale under voltage (nonequilibrium) and milestoning without voltage (equilibrium) - provided complementary information that allowed detailed analysis of the kinetics of cAMP transit in the absence and presence of voltage. The relation between voltage simulations and milestoning simulations were investigated by deriving 
the PMF under voltage from the intrinsic PMF, which revealed how mobile ions and protein dipole contribute to the resulting free energy landscape. In addition, unbiased simulations within each milestoning cell allowed us to examine the dipole orientation of cAMP through the pore, with and without voltage, and the short-range and long-range effects of cAMP on pore width. featured a prominent intra-pore binding site, characterized by a high density of positive charge in a wide entrance region of the pore. The PMF and MFPT from milestoning further allow us to estimate the binding constants, dissociation and association rates of cAMP inside the channel. Because this site directly communicates with the bulk aqueous compartment, the "saturability" of this site is expected to be more complex than for a single-occupancy ion binding site in a narrow pore. The results also suggest that while more than one cAMP can be within the pore, this multiple occupancy only occurs at this wide binding site, and that there is single cAMP occupancy in the rest of the pore. Thus, we do not anticipate for cAMP the types of multi-occupancy effects seen in many ion channels that involve interactions between sequential single-occupancy binding sites in the permeation path.

At the cAMP concentration used in the voltage simulations, we noted that there were selfinteractions among the cAMP molecules at the pore entrance (and not in bulk); cAMP molecules interacted with each other via pi-stacking and hydrogen bonding. This was likely due to the 26.5 $\mathrm{mM}$ bulk concentration of cAMP used to obtain a measurable number of transits during the voltage

517 simulation, which was greatly increased at the pore entrance. Although $\mathrm{Mg}^{2+}$ ions were included 518 in all simulations, they did not play a role in cAMP clustering or permeation. The cAMP self519 interactions are unlikely to occur at any physiological cAMP concentrations. However, this finding 520 does point out a caution when a high concentration of ligands is used in computations to speed up 521 the sampling.

While the cAMP binding sites and barriers are consistent between nonequilibrium 523 simulation and milestoning simulation, the kinetic features are different. Under equilibrium 524 simulation, the cAMP transit time is $\sim 3$ times faster inward than outward. This asymmetric rate is not seen under voltage simulations. By exploring how voltages influence the permeation free

526 energy, we found this is likely due to the negative voltage reducing the free energy barrier for 527 outward cAMP flux. The influence of the voltage of the free energy profile of cAMP permeation was estimated using intrinsic PMF from milestoning and the electrostatic potential change induced 
by the electric field. The PMF profiles under the two opposite voltages highlight how the external

530 voltage alters the thermodynamics and kinetics of cAMP permeation by changing both the relative

531 free energy as well as free energy barriers. One feature that emerged from this investigation was

532 the recognition of that for a wide pore such as connexin26, the effect of voltage on the mobile

533 charges and polarizable elements within the pore produces changes in the electrostatic field within

534 the pore that affect permeation. These voltage-induced modifications of the reaction field alter the

535 energetic landscape in protein-specific ways. For the connexin channel, these changes are imposed

536 on the intrinsic dipole within the pore, which is responsible for the asymmetric effect of symmetric

537 voltage changes on the overall PMF.

538 The primary impact of this work will be to establish a way to generate meaningful

539 hypotheses/understanding about the basis of molecular selectivity of connexin channels and to

540 understand how mutations of connexin proteins alter the selectivity and thereby cause human

541 pathologies. Such hypotheses can be tested experimentally and computationally in a synergistic

542 manner. The broader application will be studies of permeation of other biological molecules (e.g.,

543 ATP, glutathione, IP3) known to permeate Cx26 channels, and eventually to extend the work to

544 other connexin isoforms as validated atomic models are developed. The methods presented in this

545 study can be applied to understand the molecular permeation through a large pore in general. Input

546 files and raw data used to generate each figure, as well as python3 scripts for milestoning analysis

547 are publicly available at https://github.com/LynaLuo-Lab/Connexin-cAMP-milestoning. Long

548 timescale MD trajectories are publicly available on Anton2 supercomputer.

551 Methods

\section{Force Field and cAMP Parameterization}

553 CHARMM36 force field was used for protein(27, 28), POPC lipids(29), KCl, and TIP3P water 554 (30). $\mathrm{Mg}^{2+}$ parameters were from Yoo and $\operatorname{Aksimentiev(31),~in~which~the~van~der~Waals~}$ 555 interaction parameters were fine-tuned to reproduce experimental osmotic pressure. For cAMP, 556 force field parameters were first generated from CHARMM CGenFF(32). Additional dihedral 557 fitting between imidazole and pyran groups in the cAMP structure was performed in VMD ffTK 
plugin(33). The final optimized cAMP parameters are provided in Table $\mathbf{S 3}$ and https://github.com/LynaLuo-Lab/Connexin-cAMP-milestoning.

\section{System Setup and Equilibrium Protocol}

562 The atomistic model of Cx26 was taken from our previous work( 6 ). The system of Cx 26 embedded in a solvated 1-palmitoyl-2-oleoylphosphatidylcoline (POPC) bilayer with ions and TIP3P water molecules was built and equilibrated following the step-by-step protocol used in Membrane Builder in CHARMM-GUI website $(34,35)$. The terminal amino acid of each segment was capped using acetylated N-terminus (ACE) and methylated C-terminus (CT1), and three disulfide bonds were added between the residue pairs of C53 and C180, C64 and C169, C60 and C174 respectively per protomer, so the entire channel contained a total of 18 disulfide bonds, consistent with the original crystal structure (PDB ID 2ZW3). Two systems containing a single cAMP for milestoning simulations and 27 cAMP for nonequilibrium simulations were constructed (see Table S1 for system details for Milestoning simulation and Anton2 simulation). The system was energy minimized and serially equilibrated in NVT and NPT ensembles with positional restraints using AMBER18(36). Temperature was maintained at 310.15 K using Langevin thermostat (37, 38) and $1 \mathrm{~atm}$ was maintained by Monte Carlo barostat pressure control $(39,40)$. The time step was 2 fs.

575 Cutoff for calculating van der Waals interactions and short-range electrostatic interactions was set at $12 \AA$ and force-switched at $10 \AA$. Long-range electrostatic interactions were calculated using the particle mesh Ewald algorithm (41).

\section{Anton2 Simulation Protocol}

After 35 ns equilibrium simulation, the system was run on Anton2 supercomputer with 2.0 fs timestep. Lennard-Jones interactions were truncated at 11-13 $\AA$ and long-range electrostatics were evaluated using the k-Gaussian Split Ewald method (42). Pressure regulation was accomplished via the Martyna-Tobias-Klein (MTK) barostat, to maintain 1 bar of pressure, with a tau parameter of $0.0416667 \mathrm{ps}$ and reference temperature of $310.15 \mathrm{~K}$. The barostat period was set to the default value of 480 ps per timestep. Temperature control was accomplished via the NoséHoover thermostat with the same tau parameter. The mts parameter was set to 4 timesteps for the

587 barostat control and 1 timestep for the temperature control. The thermostat interval was set to the default value of 24 ps per timestep. A $600 \mathrm{~ns}$ equilibrium simulation was finished before applying 
voltage. Constant electric fields of -200 or $+200 \mathrm{mV}$ respectively were added for $2 \mu$ s simulation time with trajectories saved every 200 ps.

\section{Milestoning MD Simulation Setup}

The AMBER18 CUDA version currently does not support Cartesian coordinates as a collective variable. Thus, we pinned two water molecules using high Cartesian restrain with 6000

$595 \mathrm{kcal} \mathrm{mol}^{-1} \AA^{-2}$ force constant in the top and bottom bulk region (20 $\AA$ away from the intracellular

596 entrance and $35 \AA$ away from the extracellular entrance of the channel). We then used the projected distance on the $\mathrm{z}$ vector between the nearest pinned water oxygen and center of mass of cAMP to define the Voronoi cells along the z-axis. A cylindrical restraint with a radius of $30 \AA$ was applied to cAMP to confine the sampling in the bulk region. To prevent protein drifting, a strong harmonic distance restraint with force constant $2000 \mathrm{kcal} \mathrm{mol}^{-1} \AA^{-2}$ along $x y z$-axes between the fixed water oxygen in intracellular bulk and the center of mass of the protein was added. The simulation protocol in AMBER18 is the same as above, except all milestoning simulations were run in NVT ensemble. The timestep was $2.0 \mathrm{fs}$, and each trajectory was saved every 500 frames. Confinement within the Voronoi cells was obtained using flat-harmonic restraint with force constant of $100 \mathrm{kcal}$ $\mathrm{mol}^{-1} \AA^{-2}$.

\section{Convergence of PMF and MFPT}

A total of 53 Voronoi cells $B_{i}$ are evenly distributed $2 \AA$ apart along the z-axis, and a milestone state $S_{i j}$ is defined as the boundary between two adjacent Voronoi cells $B_{i}$ and $B_{j}$. If $k_{i \rightarrow j}$ is the rate of attempted escape from cells $B_{i}$ to $B_{j}$, since at statistical equilibrium the total flux in and out of each cell is zero, thus the equilibrium probability $\pi_{i}$ for the system to be in cell $B_{i}$ satisfies a balance equation:

$$
\sum_{j=1, j \neq i} \pi_{j} k_{j \rightarrow i}=\sum_{j=1, j \neq i} \pi_{i} k_{i \rightarrow j,} \sum_{i=1} \pi_{i}=1
$$


618 rate of attempted escape on both sides of the cell $B_{i}$, called $k_{i \rightarrow j}$ and $k_{i \rightarrow k}$, as well as the retention

619 rate inside each cell $B_{i}$ over time (Figure 9a).

$620 \quad$ By defining a milestone $S_{i j}$ as the boundary between two adjacent Voronoi cells $B_{i}$ and $B_{j}$,

621 the dynamics of the system is reduced to that of a Markov chain in the state space of the milestones

622 indices $(9)$. The MFPT between any pair of milestones $S_{i j}$ and $S_{i \mathrm{k}}$ can hence be calculated from the

623 rate matrix whose elements $q_{i j, i k}$, the rate of moving from milestone $S_{i j}$ to $S_{i \mathrm{k}}$, are given by:

$$
q_{i j, i k}=\frac{\pi_{i} n_{i j, i k}^{i}}{\pi_{i} r_{i j}^{i}+\pi_{j} r_{i j}^{j}}
$$

626 where $n_{i j, i k}^{i}$ is the number of transitions from $S_{i j}$ to $S_{i \mathrm{k}}$, normalized by the time spend in cell $B_{i}$,

627 and $r_{i j}^{i}$ is the time passed in cell $B_{i}$ after having hit $S_{i j}$ before hitting any other milestone,

628 normalized by the total time spent in cell $B_{i}$. Therefore, after the convergence of $\pi_{i}$, the 629 convergence of MFPT can be monitored directly from the accumulated $n_{i j, i k}^{i}$ and $r_{i j}^{i}$ (Figure 9b).

630 The final $10 \mathrm{~ns}$ windowed relative RMSD is also given at the top of each panel as a measure of the 631 degree of convergence for the corresponding rate matrix entry components. This relative RMSD

632 within 5\% (averaged over all replicas) is used as convergence criteria for all Voronoi cells, and in 633 most cases, RMSD is below $2 \%$ of the mean value. 
a

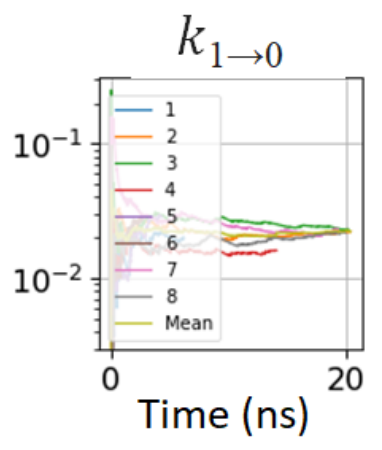

b

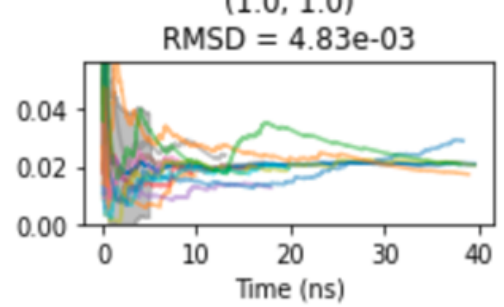

$(3.0,1.0)$

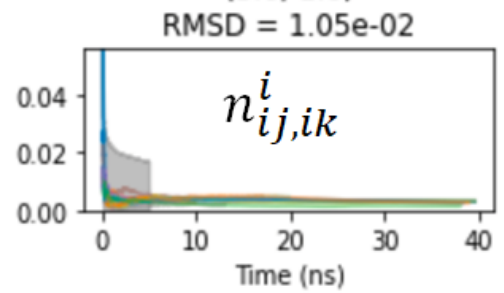

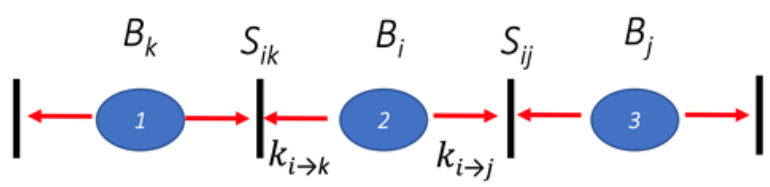

638

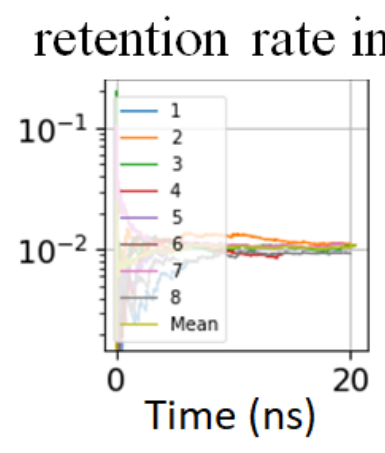

$(1.0,2.0)$

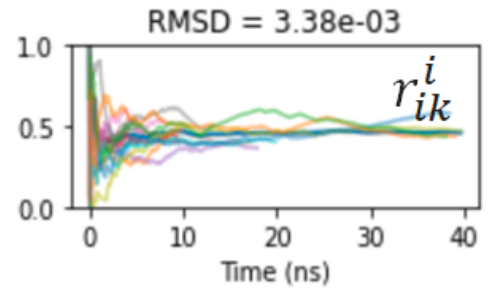

$(3.0,2.0)$

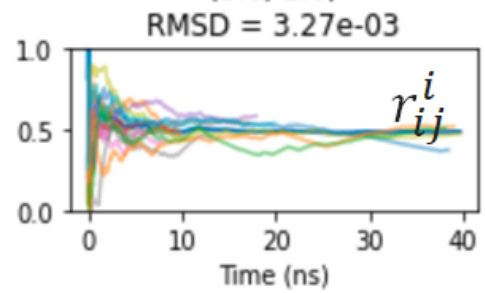

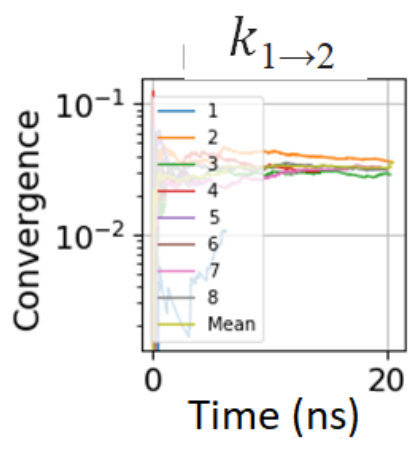

$(1.0,3.0)$

RMSD $=1.03 e-02$

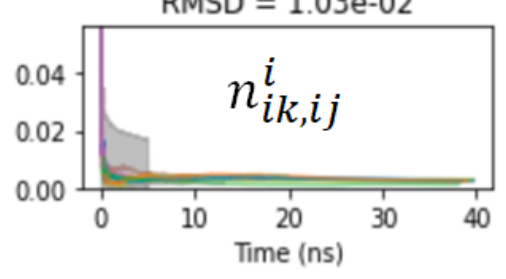

$(3.0,3.0)$

RMSD $=7.68 \mathrm{e}-03$

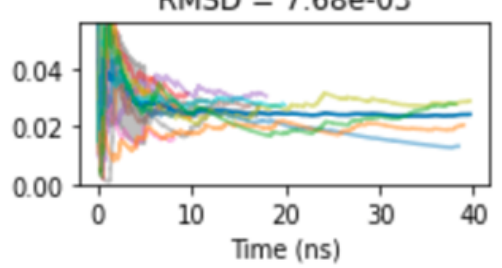

Figure 9. a) PMF convergence plots of a Voronoi cell for index $01\left(B_{1}\right)$ over time. The left and right plots represent the probability of the attempted escape to the left or right milestone states, $k_{1 \rightarrow 0}$ and $k_{1 \rightarrow 2}$. The middle plot is the retention rate inside the cell. Colors represent different replicas and the mean of all the replicas. b) convergence plots for MFPT for milestoning cell index $2\left(B_{2}\right)$. The upper right and lower left panels correspond to the frequencies of transitions from cell 1 to cell 3 , and from cell 3 to cell 1 , respectively $\left(n_{i j, i k}^{i}\right.$ and $\left.n_{i k, i j}^{i}\right)$. The upper and lower center panels correspond to the percentage of time spent in cell 2 after last touching cell 1 and $3\left(r_{i j}^{i}\right)$, respectively. The other two entries are not used in analysis directly but would correspond to re-entering cell 1 or 3 after last visiting that same cell previously. The final $10 \mathrm{~ns}$ (last 10,000 frames) windowed relative RMSD is also given at the top of each panel as a measure of the degree of convergence for the corresponding rate matrix entry components. The convergence plots of all 53 cells are available on Github repository https://github.com/LynaLuo-Lab/Connexin-cAMP-milestoning. 


\section{ACKNOWLEDGMENTS}

This work was supported by NIH Grant R01-GM130834 and WesternU intramural grant. Computational

655 resources were provided via the Extreme Science and Engineering Discovery Environment (XSEDE)

656 allocation TG-MCB160119, which is supported by NSF grant number ACI-154862. Anton2 computer time

657 was provided by the Pittsburgh Supercomputing Center (PSC) through NIH Grant R01-GM116961. The

658 Anton2 machine at PSC was generously made available by D.E.Shaw Research.

\section{AUTHOR CONTRIBUTIONS}

W.J. prepared the system, re-parameterized cAMP force field, and performed nonequilibrium simulations. Y-C.L. performed milestoning simulations. W.J and Y-C.L analyzed both simulation results. W.M.B-S. prepared milestoning analysis scripts in python3 and performed convergence analysis. W.M.B-S. and L.M. supervised milestoning simulation. Y.L.L, A.H, L.M, and J.C designed the project and wrote the paper with 664 input from all authors.

\section{REFERENCES}

1. A. L. Harris, Connexin channel permeability to cytoplasmic molecules. Prog Biophys Mol Biol 94, 120-143 (2007).

2. A. L. Harris, Emerging issues of connexin channels: biophysics fills the gap. $Q$ Rev Biophys 34, 325-472 (2001).

3. A. Pfenniger, A. Wohlwend, B. R. Kwak, Mutations in connexin genes and disease. Eur J

4. I. E. García, P. Prado, A. Pupo, O. Jara, D. Rojas-Gómez, P. Mujica, C. Flores-Muñoz, J.

5. A. L. Harris, Connexin specificity of second messenger permeation: real numbers at last. $J$

6. Y. Luo, A. R. Rossi, A. L. Harris, Computational studies of molecular permeation through connexin26 channels. Biophys J 110, 584-599 (2016).

7. A. K. Faradjian, R. Elber, Computing time scales from reaction coordinates by milestoning. J Chem Phys 120, 10880-10889 (2004).

8. R. Elber, Milestoning: An Efficient Approach for Atomically Detailed Simulations of Kinetics in Biophysics. Annu Rev Biophys 49, 69 (2020).

9. E. Vanden-Eijnden, M. Venturoli, Markovian milestoning with Voronoi tessellations. J Chem Phys 130, 194101 (2009). 
10. T.-Q. Yu, M. Lapelosa, E. Vanden-Eijnden, C. F. Abrams, Full kinetics of CO entry, internal diffusion, and exit in myoglobin from transition-path theory simulations. J Am Chem Soc 137, 3041-3050 (2015).

11. B. R. Jagger, A. A. Ojha, R. E. Amaro, Predicting Ligand Binding Kinetics Using a Markovian Milestoning with Voronoi Tessellations Multiscale Approach. J Chem Theory Comput 16, 5348-5357 (2020).

12. L. Maragliano, E. Vanden-Eijnden, B. Roux, Free energy and kinetics of conformational transitions from Voronoi tessellated milestoning with restraining potentials. J Chem Theory Comput 5, 2589-2594 (2009).

13. X. He, Y. Shen, F. R. Hung, E. E. Santiso, Heterogeneous nucleation from a supercooled ionic liquid on a carbon surface. J Chem Phys 145, 211919 (2016).

14. G. Alberini, F. Benfenati, L. Maragliano, Molecular dynamics simulations of ion selectivity in a claudin-15 paracellular channel. J Phys Chem B 122, 10783-10792 (2018).

15. G. Kanaporis, G. Mese, L. Valiuniene, T. W. White, P. R. Brink, V. Valiunas, Gap junction channels exhibit connexin-specific permeability to cyclic nucleotides. J Gen Physiol 131, 293-305 (2008).

16. V. K. Verselis, C. S. Ginter, T. A. Bargiello, Opposite voltage gating polarities of two closely related onnexins. Nature 368, 348-351 (1994).

17. B. I. Pinto, I. E. García, A. Pupo, M. A. Retamal, A. D. Martínez, R. Latorre, C. González, Charged residues at the first transmembrane region contribute to the voltage dependence of the slow gate of connexins. J Biol Chem 291, 15740-15752 (2016).

18. H. C. Berg, Random Walks in Biology: New and Ezpanded Edition. (Princeton, 1984).

19. V. H. Hernandez, M. Bortolozzi, V. Pertegato, M. Beltramello, M. Giarin, M. Zaccolo, S. Pantano, F. Mammano, Unitary permeability of gap junction channels to second messengers measured by FRET microscopy. Nat Methods 4, 353-358 (2007).

20. S. Berneche, B. Roux, A microscopic view of ion conduction through the $\mathrm{K}+$ channel. Proc Natl Acad Sci 100, 8644-8648 (2003).

21. B. Roux, Statistical mechanical equilibrium theory of selective ion channels. Biophys $J$ 77, 139-153 (1999).

22. B. Roux, The membrane potential and its representation by a constant electric field in computer simulations. Biophys J 95, 4205-4216 (2008).

23. J. Gumbart, F. Khalili-Araghi, M. Sotomayor, B. Roux, Constant electric field simulations of the membrane potential illustrated with simple systems. Biochim Biophys Acta 1818, 294302 (2012).

24. A. Aksimentiev, K. Schulten, Imaging $\alpha$-hemolysin with molecular dynamics: ionic conductance, osmotic permeability, and the electrostatic potential map. Biophys $J \mathbf{8 8}$, 3745-3761 (2005).

25. T. Paramo, A. East, D. Garzón, M. B. Ulmschneider, P. J. Bond, Efficient characterization of protein cavities within molecular simulation trajectories: trj_cavity. J Chem Theory Comput 10, 2151-2164 (2014).

26. T. Kwon, A. L. Harris, A. Rossi, T. A. Bargiello, Molecular dynamics simulations of the Cx26 hemichannel: evaluation of structural models with Brownian dynamics. J Gen Physiol 138, 475-493 (2011). 
27. A. D. MacKerell Jr, D. Bashford, M. Bellott, R. L. Dunbrack Jr, J. D. Evanseck, M. J. Field, S. Fischer, J. Gao, H. Guo, S. Ha, D. Joseph-McCarthy, L. Kuchnir, K. Kuczera, T. K. Lau, C. Mattos, S. Michnick, T. Ngo, D. T. Nguyen, R. Stote, J. Straub, M. Watanabe, J. Wiorkiewicz-Kuczera, D. Yin, M. Karplus, All-atom empirical potential for molecular modeling and dynamics studies of proteins. J Phys Chem B 102, 3586-3616 (1998).

28. A. D. Mackerell Jr, M. Feig, C. L. Brooks III, Extending the treatment of backbone energetics in protein force fields: Limitations of gas-phase quantum mechanics in reproducing protein conformational distributions in molecular dynamics simulations. J Comput Chem 25, 1400-1415 (2004).

29. J. B. Klauda, R. M. Venable, J. A. Freites, J. W. O'Connor, D. J. Tobias, C. MondragonRamirez, I. Vorobyov, A. D. MacKerell Jr, R. W. Pastor, Update of the CHARMM all-atom additive force field for lipids: validation on six lipid types. J Phys Chem B 114, 7830-7843 (2010).

30. W. L. Jorgensen, J. Chandrasekhar, J. D. Madura, R. W. Impey, M. L. Klein, Comparison of simple potential functions for simulating liquid water. J Chem Phys 79, 926-935 (1983).

31. J. Yoo, A. Aksimentiev, Improved parametrization of $\mathrm{Li}+, \mathrm{Na}+, \mathrm{K}+$, and $\mathrm{Mg} 2+$ ions for allatom molecular dynamics simulations of nucleic acid systems. J Phys Chem Lett 3, 45-50 (2012).

32. K. Vanommeslaeghe, E. Hatcher, C. Acharya, S. Kundu, S. Zhong, J. Shim, E. Darian, O. Guvench, P. Lopes, I. Vorobyov, A. D. MacKerell Jr, CHARMM general force field: A force field for drug-like molecules compatible with the CHARMM all-atom additive biological force fields. J Comput Chem 31, 671-690 (2010).

33. C. G. Mayne, J. Saam, K. Schulten, E. Tajkhorshid, J. C. Gumbart, Rapid parameterization of small molecules using the force field toolkit. J Comput Chem 34, 2757-2770 (2013).

34. S. Jo, T. Kim, W. Im, Automated builder and database of protein/membrane complexes for molecular dynamics simulations. PloS one 2, e880 (2007).

35. S. Jo, T. Kim, V. G. Iyer, W. Im, CHARMM-GUI: a web-based graphical user interface for CHARMM. J Comput Chem 29, 1859-1865 (2008).

36. D. Case, D. Cerutti, T. Cheatham, T. Darden, R. Duke, T. Giese, H. Gohlke, A. Goetz, D. Greene, N. J. U. o. C. Homeyer, AMBER reference manual. (2018).

37. W. G. Hoover, A. J. Ladd, B. Moran, High-strain-rate plastic flow studied via nonequilibrium molecular dynamics. Phys Rev Lett 48, 1818 (1982).

38. D. J. Evans, Computer "experiment"for nonlinear thermodynamics of Couette flow. J Chem Phys 78, 3297-3302 (1983).

39. J. Åqvist, P. Wennerström, M. Nervall, S. Bjelic, B. O. Brandsdal, Molecular dynamics simulations of water and biomolecules with a Monte Carlo constant pressure algorithm. Chem. Phys. Lett 384, 288-294 (2004).

40. M. P. Allen, D. J. Tildesley, Computer simulation of liquids. (Oxford university press, 2017).

41. T. Darden, D. York, L. Pedersen, Particle mesh Ewald: An N· log (N) method for Ewald sums in large systems. J Chem Phys 98, 10089-10092 (1993).

42. Y. Shan, J. L. Klepeis, M. P. Eastwood, R. O. Dror, D. E. Shaw, Gaussian split Ewald: A fast Ewald mesh method for molecular simulation. J Chem Phys 122, 054101 (2005). 


\section{Supporting Information}

Figure S1. Pore radius profiles from two simulations under $+/-200 \mathrm{mV}$, calculated using Hole
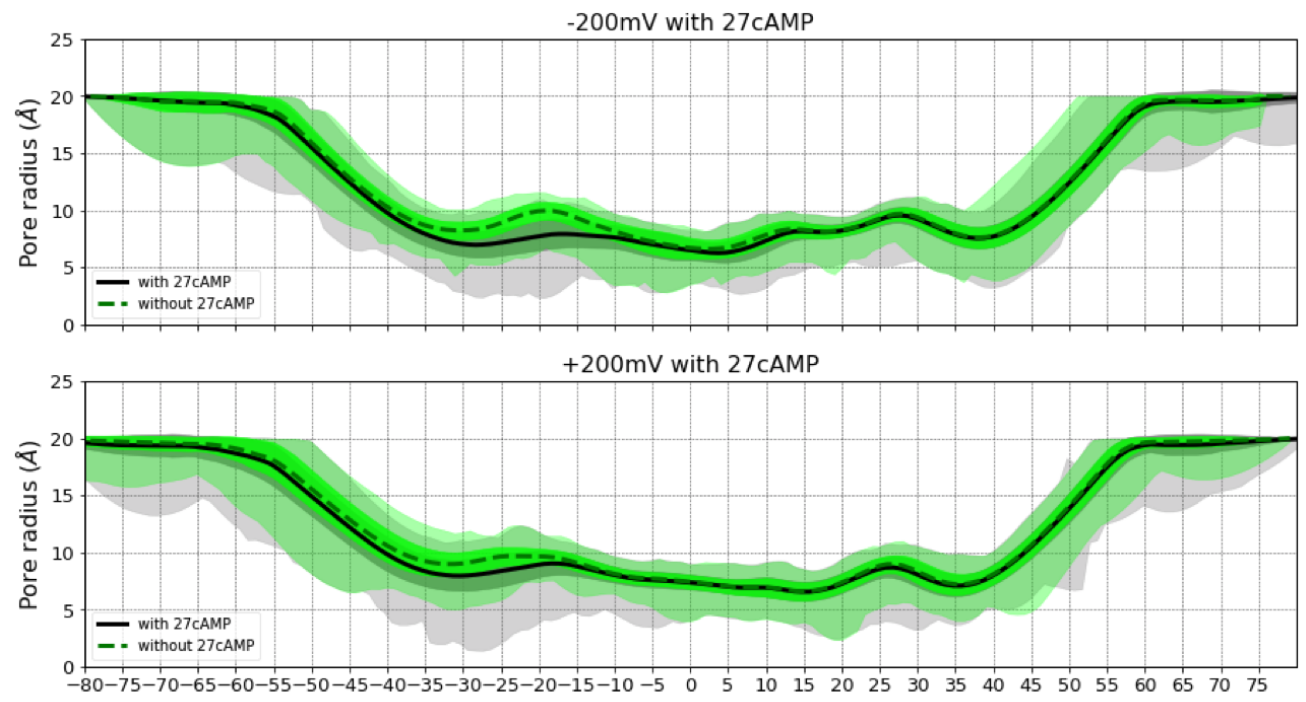
program. The black line is the average value over last $1 \mu \mathrm{s}$, with $1.2 \mathrm{~ns}$ interval between snapshots. minimum and maximum radius values. The dashed line and green shade are the pore radius profile

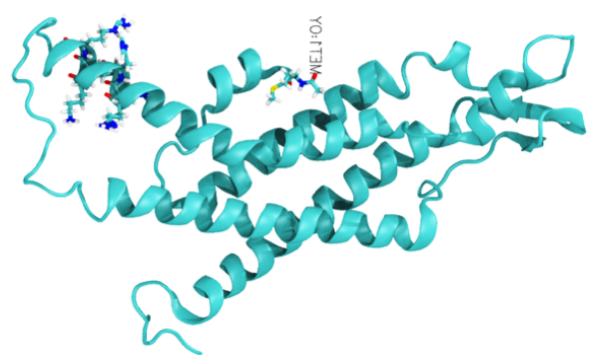
with protein only. 
bioRxiv preprint doi: https://doi.org/10.1101/2021.01.18.427208. this version posted January 192021 . The copyright holder for this preprint (which was not certified by peer review) is the author/funder, who has granted bioRxiv a license to display the preprint in perpetuity. It is made available under aCC-BY-ND 4.0 International license.

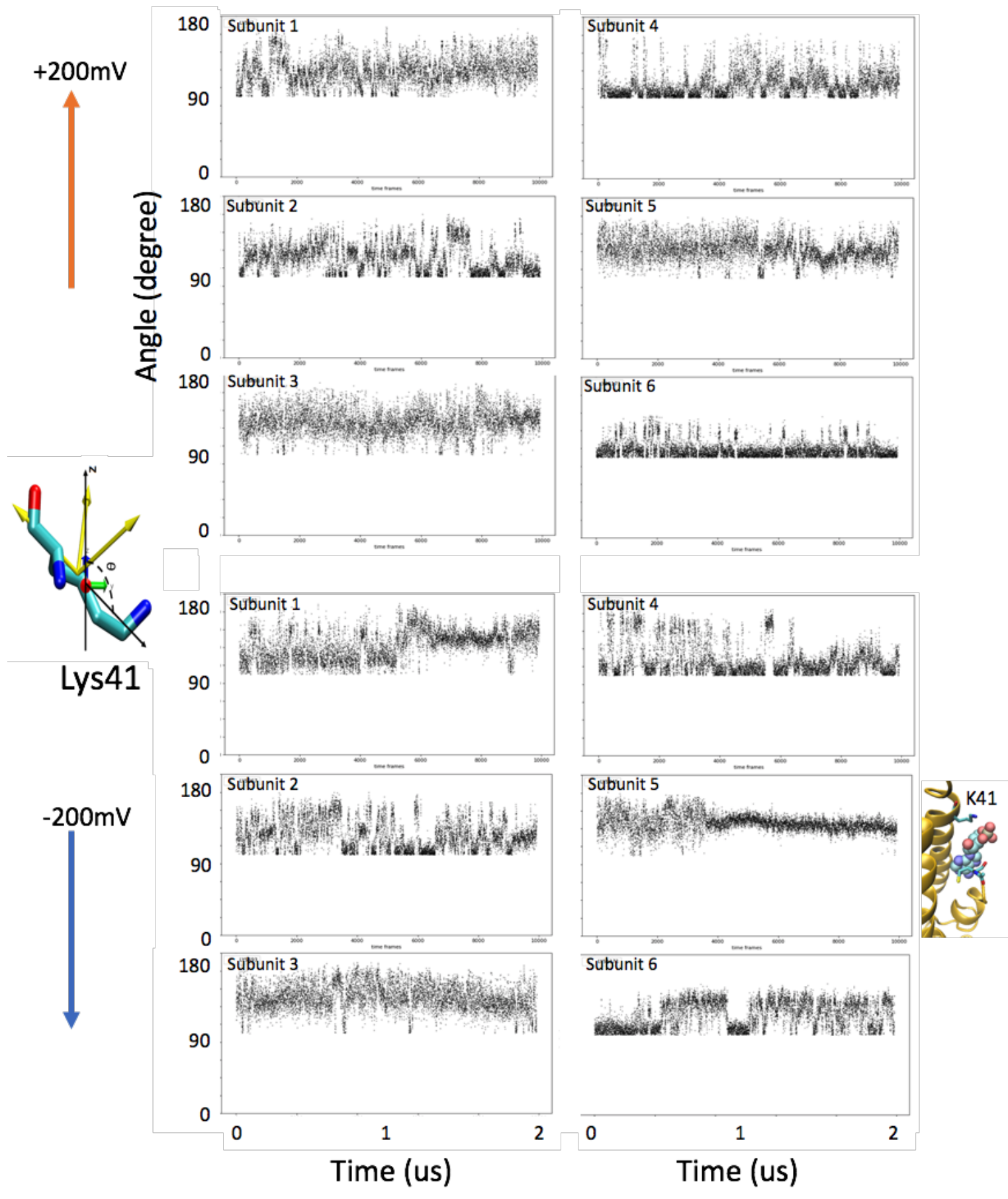

Figure S2a. Fluctuation of the angles between the principal axis of Lys41 and z-vector in each subunit under $+/-200 \mathrm{mV}$. Representative Lys residue shown in the licorice model with its principal axes and the angle plotted. 


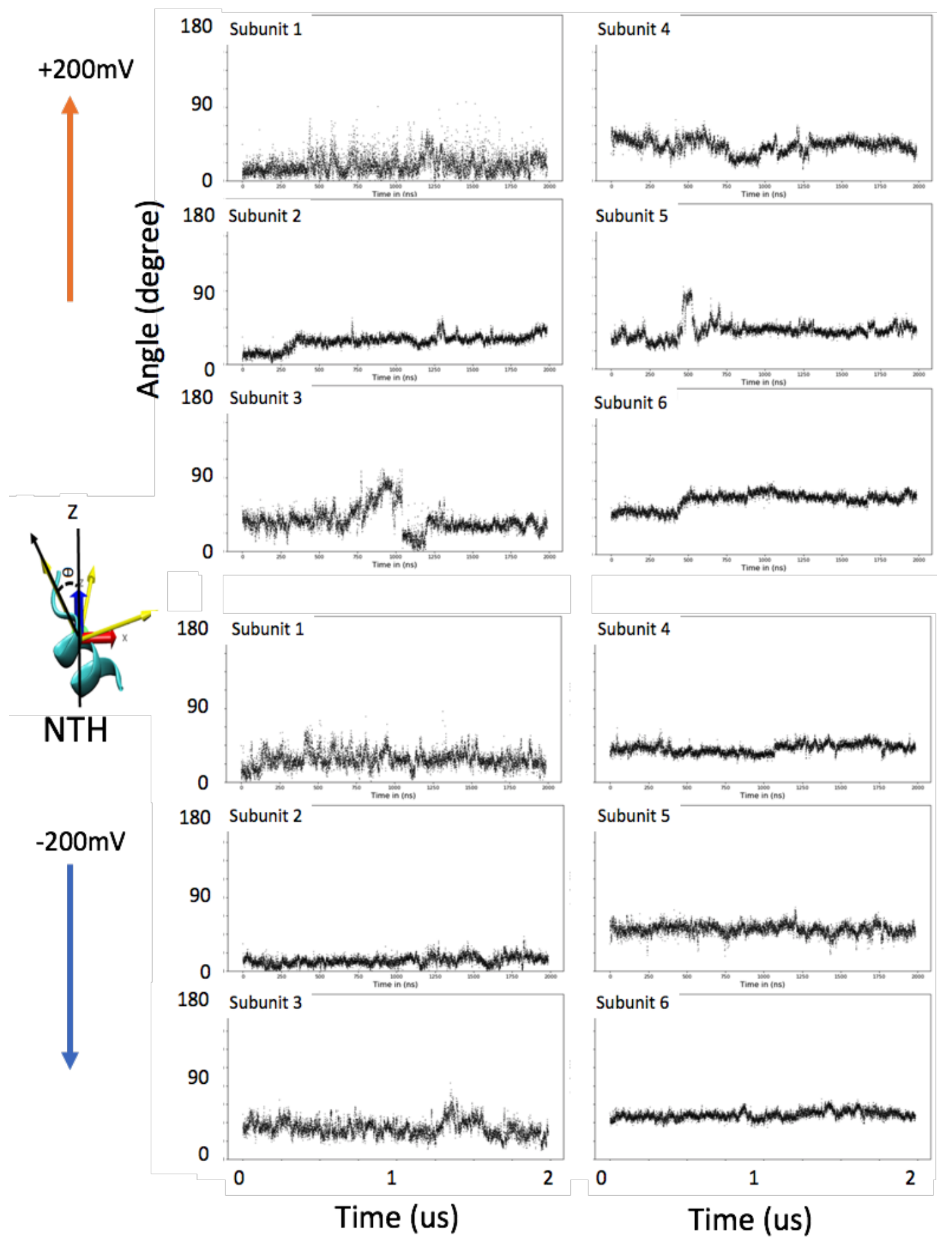

Figure S2b. Fluctuation of the angles between the principal axis of NTH (1-11) and z-vector in each subunit under $+/-200 \mathrm{mV}$. Representative NTH shown in the new cartoon model with its principal axes and the angle plotted. 
807 Table S1. Description of the computational systems

\begin{tabular}{|c|c|c|c|c|c|c|c|c|}
\hline & & & & & & Numl & (concentration & \\
\hline & & ing & & $\left(\mathrm{A}^{3}\right)$ & atoms & cAMP & $\mathrm{K}+/ \mathrm{Mg} 2+/ \mathrm{Cl}-$ & POPC/water \\
\hline Anton2 & $\begin{array}{l}+/- \\
200 \mathrm{mV}\end{array}$ & $\begin{array}{l}\text { ACE } \\
\text { CT1 }\end{array}$ & $\begin{array}{l}\text { C53-C180 } \\
\text { C60-C174 } \\
\text { C64-C169 }\end{array}$ & $\begin{array}{l}122 x \\
122 x \\
154\end{array}$ & 237030 & $\begin{array}{l}27 \\
(26.5 \\
\mathrm{mM})\end{array}$ & $\begin{array}{l}82 / 27 / 163 \\
(80 / 26 / 160 \\
\mathrm{mM})\end{array}$ & $323 / 56569$ \\
\hline Milestoning & $0 \mathrm{mV}$ & $\begin{array}{l}\text { ACE } \\
\text { CT1 }\end{array}$ & $\begin{array}{l}\text { C53-C180 } \\
\text { C60-174 } \\
\text { C64-C169 }\end{array}$ & $\begin{array}{l}121 \mathrm{x} \\
121 \mathrm{x} \\
155\end{array}$ & 234652 & $\begin{array}{l}1 \\
(1 \mathrm{mM})\end{array}$ & $\begin{array}{l}82 / 1 / 137 \\
(81 / 1 / 135 \\
\mathrm{mM})\end{array}$ & $324 / 56191$ \\
\hline
\end{tabular}

*Concentrations were calculated based on number of water molecules.

Table S2. Transition time and dwell time (ns) from $2 \mu$ s trajectories at $+200 \mathrm{mV}$ and -200

$812 \mathbf{m V}$.

\begin{tabular}{|c|c|c|c|c|c|c|}
\hline \multirow[b]{2}{*}{$\begin{array}{c}\text { Transition } \\
\text { Event }\end{array}$} & \multicolumn{3}{|c|}{$+200 \mathrm{mV}$ (inward flux) } & \multicolumn{3}{|c|}{$-200 \mathrm{mV}$ (outward flux) } \\
\hline & $\begin{array}{c}\text { Transition Time } \\
-\mathbf{5 0}<\mathrm{Z}<50\end{array}$ & $\begin{array}{l}\text { Dwell time } \\
-50<z<-20\end{array}$ & $\begin{array}{c}\text { Barrier } \\
\text { crossing } \\
-20<\mathbf{Z}<50\end{array}$ & $\begin{array}{c}\text { Transition Time } \\
-\mathbf{5 0}<\mathbf{Z}<\mathbf{5 0}\end{array}$ & $\begin{array}{c}\text { Dwell time } \\
-50<Z<-20\end{array}$ & $\begin{array}{c}\text { Barrier } \\
\text { crossing } \\
-20<\mathbf{z}<50\end{array}$ \\
\hline 1 & 222 & 24 & 198 & 160 & 110 & 50 \\
\hline 2 & 1218 & 1058 & 160 & 242 & 210 & 32 \\
\hline 3 & 416 & 308 & 108 & 290 & 50 & 240 \\
\hline 4 & 282 & 248 & 34 & 1496 & 1400 & 96 \\
\hline 5 & 542 & 498 & 44 & 230 & 162 & 68 \\
\hline 6 & 290 & 149 & 140 & 630 & 570 & 60 \\
\hline 7 & 192 & 120 & 72 & 384 & 350 & 34 \\
\hline 8 & 454 & 222 & 232 & 152 & 100 & 52 \\
\hline 9 & 426 & 150 & 276 & 1410 & 1228 & 182 \\
\hline 10 & & & & 198 & 76 & 122 \\
\hline 11 & & & & 390 & 150 & 240 \\
\hline 12 & & & & 445 & 350 & 95 \\
\hline Raw mean & 449 & 308 & 140 & 502 & 396 & 105 \\
\hline Sample mean* & 448 & 305 & 143 & 510 & 398 & 106 \\
\hline $95 \%$ CI* & $204-1646$ & 149-940 & $79-330$ & $249-1571$ & $205-1086$ & $54-290$ \\
\hline
\end{tabular}

$813 *$ The sample mean and confidence intervals are based on Maximum Likelihood Estimate by fitting the

814 exponential distributions (see code at https://github.com/LynaLuo-Lab/MD-data-uncertainty-

815 analysis/blob/master/confidence_interval_exponential.ipynb).

816

817

818

Table S3. Optimized cgenff force field parameters for cAMP

\begin{tabular}{|l|l|r|r|r|r|r|}
\hline \multicolumn{7}{|c|}{ BONDS } \\
\hline CG3RC1 & OG3R60 & 334.3 & 1.411 & & & \\
\hline OG3R60 & PG1 & 237 & 1.61 & & & \\
\hline
\end{tabular}




\begin{tabular}{|c|c|c|c|c|c|c|}
\hline \multicolumn{7}{|c|}{ ANGLES } \\
\hline CG3RC1 & CG321 & OG3R60 & 75.7 & 110.1 & & \\
\hline CG321 & CG3RC1 & OG3C51 & 45 & 111.5 & & \\
\hline CG3C51 & CG3RC1 & OG3R60 & 58 & 106.5 & 8 & 2.561 \\
\hline CG3RC1 & CG3RC1 & OG3R60 & 53.35 & 111 & 8 & 2.561 \\
\hline OG3R60 & CG3RC1 & HGA1 & 45.9 & 108.5 & & \\
\hline CG3C51 & OG3C51 & CG3RC1 & 170 & 109 & & \\
\hline CG321 & OG3R60 & PG1 & 20 & 120 & 35 & 2.33 \\
\hline CG3RC1 & OG3R60 & PG1 & 20 & 120 & 35 & 2.33 \\
\hline OG2P1 & PG1 & OG3R60 & 98.9 & 107.5 & & \\
\hline OG3R60 & PG1 & OG3R60 & 80 & 104.3 & & \\
\hline \multicolumn{7}{|c|}{ DIHEDRALS } \\
\hline OG3R60 & CG321 & CG3RC1 & CG3RC1 & 0.6 & 1 & 0 \\
\hline OG3R60 & CG321 & CG3RC1 & CG3RC1 & 0.45 & 2 & 0 \\
\hline OG3R60 & CG321 & CG3RC1 & CG3RC1 & 0.7 & 3 & 0 \\
\hline OG3R60 & CG321 & CG3RC1 & OG3C51 & 3.4 & 1 & 180 \\
\hline OG3R60 & CG321 & CG3RC1 & HGA1 & 0.195 & 3 & 0 \\
\hline HGA2 & CG321 & CG3RC1 & OG3C51 & 0.16 & 3 & 0 \\
\hline CG3RC1 & CG321 & OG3R60 & PG1 & 0.002 & 1 & 180 \\
\hline CG3RC1 & CG321 & OG3R60 & PG1 & 1.14 & 2 & 180 \\
\hline CG3RC1 & CG321 & OG3R60 & PG1 & 0.086 & 3 & 0 \\
\hline HGA2 & CG321 & OG3R60 & PG1 & 0.599 & 3 & 0 \\
\hline CG3RC1 & CG3C51 & CG3C51 & NG2R51 & 0 & 3 & 0 \\
\hline CG3RC1 & CG3C51 & CG3C51 & OG3C51 & 0 & 3 & 0 \\
\hline CG3C51 & CG3C51 & CG3RC1 & OG3R60 & 2 & 3 & 180 \\
\hline CG3C51 & CG3C51 & CG3RC1 & OG3R60 & 0.4 & 5 & 0 \\
\hline CG3C51 & CG3C51 & CG3RC1 & OG3R60 & 0.8 & 6 & 0 \\
\hline OG311 & CG3C51 & CG3RC1 & OG3R60 & 0 & 3 & 0 \\
\hline HGA1 & CG3C51 & CG3RC1 & OG3R60 & 0.195 & 3 & 0 \\
\hline CG3C51 & CG3C51 & OG3C51 & CG3RC1 & 0.5 & 3 & 0 \\
\hline NG2R51 & CG3C51 & OG3C51 & CG3RC1 & 0 & 3 & 0 \\
\hline HGA1 & CG3C51 & OG3C51 & CG3RC1 & 0.3 & 3 & 0 \\
\hline CG321 & CG3RC1 & CG3RC1 & OG3R60 & 0.15 & 3 & 0 \\
\hline
\end{tabular}




\begin{tabular}{|c|c|c|c|c|c|c|}
\hline OG3C51 & CG3RC1 & CG3RC1 & OG3R60 & 1.2 & 3 & 0 \\
\hline OG3R60 & CG3RC1 & CG3RC1 & HGA1 & 0.15 & 3 & 0 \\
\hline CG321 & CG3RC1 & OG3C51 & CG3C51 & 0.3 & 3 & 0 \\
\hline CG3RC1 & CG3RC1 & OG3C51 & CG3C51 & 0 & 3 & 0 \\
\hline HGA1 & CG3RC1 & OG3C51 & CG3C51 & 0.3 & 3 & 0 \\
\hline CG3C51 & CG3RC1 & OG3R60 & PG1 & 2.553 & 1 & 180 \\
\hline CG3RC1 & CG3RC1 & OG3R60 & PG1 & 1.5 & 1 & 180 \\
\hline CG3RC1 & CG3RC1 & OG3R60 & PG1 & 0.625 & 2 & 180 \\
\hline CG3RC1 & CG3RC1 & OG3R60 & PG1 & 0.488 & 1 & 180 \\
\hline CG3RC1 & CG3RC1 & OG3R60 & PG1 & 0.972 & 3 & 180 \\
\hline HGA1 & CG3RC1 & OG3R60 & PG1 & 1.903 & 1 & 0 \\
\hline HGA1 & CG3RC1 & OG3R60 & PG1 & 0.089 & 3 & 180 \\
\hline CG321 & OG3R60 & PG1 & OG2P1 & 0.1 & 3 & 0 \\
\hline CG321 & OG3R60 & PG1 & OG3R60 & 2.248 & 1 & 180 \\
\hline CG321 & OG3R60 & PG1 & OG3R60 & 0.261 & 2 & 0 \\
\hline CG321 & OG3R60 & PG1 & OG3R60 & 2.64 & 3 & 180 \\
\hline CG321 & OG3R60 & PG1 & OG3R60 & 0.929 & 1 & 180 \\
\hline CG321 & OG3R60 & PG1 & OG3R60 & 1.149 & 3 & 0 \\
\hline CG321 & OG3R60 & PG1 & OG3R60 & 2.138 & 2 & 0 \\
\hline CG321 & OG3R60 & PG1 & OG3R60 & 1.101 & 1 & 0 \\
\hline CG321 & OG3R60 & PG1 & OG3R60 & 1.793 & 2 & 0 \\
\hline CG321 & OG3R60 & PG1 & OG3R60 & 0.54 & 3 & 0 \\
\hline CG3RC1 & OG3R60 & PG1 & OG2P1 & 0.229 & 3 & 180 \\
\hline CG3RC1 & OG3R60 & PG1 & OG3R60 & 0.799 & 1 & 0 \\
\hline CG3RC1 & OG3R60 & PG1 & OG3R60 & 0.137 & 2 & 0 \\
\hline CG3RC1 & OG3R60 & PG1 & OG3R60 & 1.1 & 3 & 0 \\
\hline
\end{tabular}

